\title{
Dissertation physico-mathématique sur les roulements du cercle
}

Père André Tacquet

\section{OpenEdition \\ Journals}

Édition électronique

URL : http://journals.openedition.org/ccibp/620

DOI : $10.4000 /$ ccibp. 620

ISSN : 2493-7460

Éditeur

Centre international Blaise Pascal

Édition imprimée

Date de publication : 5 février 1992

Pagination : 25-53

ISSN : 0249-6674

\section{Référence électronique}

Père André Tacquet, «Dissertation physico-mathématique sur les roulements du cercle », Courrier du

Centre international Blaise Pascal [En ligne], 14 | 1992, mis en ligne le 08 janvier 2016, consulté le 01

mai 2019. URL : http://journals.openedition.org/ccibp/620 ; DOI : 10.4000/ccibp.620

Ce document a été généré automatiquement le 1 mai 2019.

Centre international Blaise Pascal 


\title{
Dissertation physico-mathématique sur les roulements du cercle
}

\author{
Père André Tacquet
}

Au Lecteur

Le roulement du cercle sur un plan enferme tant de si grands paradoxes qu'on doit à juste titre le mettre au nombre des principaux secrets de la Nature. Jadis déjà Aristote a soulevé la question des cercles concentriques qui roulent ensemble, mais ni lui ni aucun autre après lui, à ma connaissance, n'a résolu la difficulté : Mais comme cette matière m'a aussi toujours semblé étonnante, plus d'une fois j'ai essayé de trouver à mon tour la sortie de ce labyrinthe, et de répandre si possible

un peu de lumière en cette difficile affaire. $\mathrm{Au}$ cours de plusieurs tentatives vaines, je suis tombé sur de nouvelles difficultés que nul n'avait remarquées : le lecteur en trouvera la synthèse dans le Scholie du premier Théorème. Enfin n'ayant jamais rien lu ni entendu qui jetât la moindre lumière dans mon embarras et mon trouble, j'ai repris tout le problème avec plus

d'application ; et ainsi j'ai fini par arriver aux réflexions formulées dans ce traité. Quelque temps après, les circonstances ont voulu que je publie sur cette matière des thèses défendues publiquement par le très illustre Comte de Hornes et de Herlies. Comme de nombreux savants ont alors approuvé cet essai, j'ai compté que le lecteur me saurait gré d'ajouter aux quatre livres sur les Cylindres et les Anneaux ce traité sur les roulements du cercle. Je 
l'ai débarrassé de la forme étroite des thèses, et j'ai développé plus amplement, surtout à la fin, des points que, pour respecter les limites du genre, j'avais résumés autant que possible dans la première édition. En outre, dans la première comme dans cette dernière édition, j'ai voulu non seulement satisfaire aux difficultés exposées dans le Scholie du premier Théorème, mais aussi exposer les autres aspects du roulement de manière qu'ils révèlent amplement leur nature

étonnante. Au revoir.

\section{Définitions}

1. Un mouvement est régulier ou uniforme lorsque dans des temps quelconques égaux sont parcourus des espaces égaux ${ }^{1}$.

2. Un mouvement est variable ou irrégulier lorsque dans des temps égaux sont parcourus des espaces inégaux.

3. On dit qu'une sphère (ou un cercle) roule sur un plan lorsqu'elle avance sur le plan de telle façon qu'elle tourne en même temps sans interruption autour de son centre. Le roulement est par conséquent composé de deux mouvements ininterrompus, savoir un mouvement circulaire et l'autre progressif ou de translation que nous appellerons souvent mouvement parallèle du centre parce que, pendant que le cercle roule, son centre décrit une ligne parallèle au plan, courbe ou droite selon l'impulsion. J'ai dit : ininterrompus. En effet si l'un ou l'autre, du mouvement de translation ou du circulaire, s'interrompt alors que l'autre continue, il n'y aura plus roulement, mais rasement ${ }^{2}$. On le montrera plus bas.

4. Il y a rasement lorsqu'un point quelconque de la circonférence passe par plus d'un seul point du plan, c'est-à-dire par une certaine partie du plan; ou lorsque plusieurs points de la circonférence (c'est-à-dire une partie de la circonférence) passent en un point du plan.

5. Un roulement est achevé lorsque le point de la circonférence qui est dans le plan au départ redescend sur ce plan.

6. J'appelle plan matériel commun celui qui comporte inégalité et aspérité ; de ce genre sont tous ceux que nous connaissons.

7. Le plan matériel parfait est complètement uniforme.

8. J'entends par plan géométrique un plan parfait, mais abstrait de la matière.

9. Lorsque, pour la brièveté, je parle d'une sphère, il faut entendre la circonférence du grand cercle de cette sphère.

10. Lorsque l'on dit infini, entendez indéfini, ou aussi grand ou nombreux que l'on veut; car c'est ainsi que parlent les géomètres.

1 Les autres termes seront définis en leur lieu, lorsque la matière le demandera.

\section{Axiomes}

1. Des vitesses égales, ou des mouvements également rapides, parcourent des espaces égaux dans des temps égaux. 
2. Une vitesse est à une autre dans le même rapport que les espaces parcourus dans le même temps. Galilée le démontre dans le Théorème II sur le mouvement uniforme ; et cela est clair par soi-même ${ }^{3}$.

\section{Théorème 1}

Lorsqu'un cercle $(\mathrm{AQ})$ roule sur un plan commun matériel, tous les cercles concentriques possibles (AP), si petits ou si grands soient-ils, s'ils se meuvent solidairement, parcourent en une rotation un espace égal à la circonférence $Q$.

Soit en effet mené à partir du centre $\mathrm{A}$ le rayon $\mathrm{ACB}$ qui coupe les cercles concentriques aux points $B$ et $C$, par lesquels on mène les tangentes $B M$ et $C L$; soit $B M$ sur un plan matériel commun, mais CL sur un plan géométrique.

Parce que par hypothèse le cercle $\mathrm{AQ}$ roule sur $\mathrm{BM}$, il est constamment tangent à la droite $\mathrm{BM}$; et par suite, pendant tout le temps du roulement, les droites conduites du centre $\mathrm{A}$ à tous les points de contact seront perpendiculaires à $\mathrm{BM}^{\mathrm{a}}$; par conséquent le rayon du cercle $\mathrm{AQ}$ sera toujours la distance du centre $\mathrm{A}$ à la droite $\mathrm{BM}$; de là, pendant tout le temps du roulement, le centre A est également distant de BM. C'est pourquoi comme CL est parallèle à $\mathrm{BM}^{\mathrm{b}}$ (en effet les angles $\mathrm{ACL}$ et $\mathrm{ABM}$ sont droits, donc égaux), le centre $\mathrm{A}$ sera toujours à égale distance de $\mathrm{CL}$, de l'intervalle du rayon $\mathrm{AC}$ ou $\mathrm{AO}$. Donc comme par hypothèse les cercles AP sont au début tangents aux droites $\mathrm{CL}$, pendant tout le temps où le cercle $\mathrm{AQ}$ tourne et touche la droite $\mathrm{BM}$, ils touchent les droites $\mathrm{CL}$; mais parce que les points $C$ sont sur la même droite, sur laquelle est le point $B$, quand, un roulement achevé, $B$ descendra de nouveau sur $B M$, les points $C$ reviendront ensemble sur leurs tangentes $\mathrm{CL}$. Donc tous les concentriques AP parcourent en un roulement des droites égales à celle que parcourt le cercle $\mathrm{AQ}$. Et $\mathrm{AQ}$ lui-même parcourt une droite égale à sa circonférence, comme l'expérience le montre, ce dont nous rendrons raison plus bas.

Un profane en géométrie ne s'en tirerait pas en disant que les cercles concentriques AP se déplacent partie en tournant, partie en rasant; que chaque point des concentriques plus petits que $\mathrm{AQ}$ est traîné sur une partie de la tangente $\mathrm{CL}$, alors que plusieurs points des concentriques plus grands que le cercle $\mathrm{AQ}$ passent par un seul et même point de la tangente. C'est absolument impossible : je le démontre ainsi.

Soit pris dans un concentrique, plus grand ou plus petit que $\mathrm{AQ}$, un point quelconque $\mathrm{O}$, par lequel du centre $A$ on mène la droite qui tombe sur le cercle déférent $A Q$ en $K$. Parce que les cercles tournent solidairement, et que les points 0 et $\mathrm{K}$ sont sur la même droite, il est évident que les points $\mathrm{O}$ et $\mathrm{K}$ tombent en même temps sur leurs plans respectifs $\mathrm{CL}$ et $\mathrm{BM}$; et qu'ils s'élèvent et s'éloignent en même temps. En effet lorsque le point $\mathrm{K}$ descendra sur BM, et deviendra le point de contact, $\mathrm{AK}$ sera perpendiculaire à la tangente $\mathrm{BM}^{\mathrm{c}}$; et par conséquent, parce que $\mathrm{BM}$ et $\mathrm{CL}$ sont équidistantes, elle sera aussi

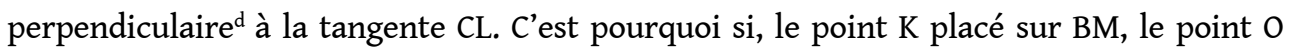
était au-dessus ou au-dessous de la droite CL, le centre A ne serait pas toujours à la même distance du rayon $\mathrm{AO}$ de la tangente $\mathrm{CL}$; on a démontré plus haut qu'il était autrement. Donc les points $O$ et $K$ atteignent et quittent ensemble leurs tangentes. C'est pourquoi lorsque le point $\mathrm{K}$ touche le plan BM dans l'instant et sans aucun rasement (car le cercle $\mathrm{AQ}$ est par hypothèse porté sur le plan matériel $\mathrm{BM}$ par un roulement véritable défini cidessus $\mathrm{e}^{\mathrm{e}}$, le point $\mathrm{O}$ touche aussi dans l'instant et sans glissement son plan géométrique CL. On montrera la même chose de n'importe quels points des circonférences concentriques PP. 
7 C'est ainsi qu'on établit la proposition géométriquement. Mais je réduis la substance de cette démonstration à la portée des philosophes comme suit. Il est impossible que le concentrique APN, qui parcourt une ligne plus grande que lui-même touche le plan par un même point de sa circonférence pendant un certain temps, autrement dit qu'il touche plusieurs points du plan, sans que le mouvement par lequel il tourne autour du centre ne s'interrompe tant soit peu. De la même façon il est impossible que le cercle ARP qui parcourt une ligne plus petite que lui, touche un certain temps le plan au même point du plan, c'est-à-dire que plusieurs points de la circonférence passent par un seul point du plan, sans que le mouvement de translation ne s'interrompe tant soit peu. Mais par hypothèse aucun des deux mouvements ne s'interrompt jamais. Donc etc. Pour voir plus clairement le sens de ce raisonnement, concevons d'abord que le petit concentrique ANP soit mû sur le plan par le seul mouvement de translation et sans aucune conversion autour du centre. Il est évident que le même point de la circonférence passe alors tangentiellement par plusieurs points du plan. Si ensuite on conçoit qu'au mouvement de translation s'ajoute une conversion du cercle autour du centre, aussi lente soit-elle, il découle évidemment que ce point de la circonférence auquel le plan est touché ne subsiste dans le plan qu'un instant seulement; en effet comme ce contact est indivisible, une conversion aussi petite qu'on veut le change, en élevant aussitôt le point qui touchait le plan. Concevons ensuite que le grand concentrique ARP soit d'abord soumis au seul mouvement de conversion autour du centre A. Il est alors évident que plusieurs points de la circonférence passent tangentiellement par le même point du plan. Si on conçoit par la suite qu'au mouvement de conversion autour du centre s'ajoute un mouvement du plan aussi petit que l'on veut vers L, de nouveau il apparait clairement que ce point du plan, qui était touché, n'est joint au cercle qui ne change pas de place que dans l'instant: en effet le contact est indivisible, si petit que soit le mouvement en avant du plan CL vers $\mathrm{L}^{4}$, il change nécessairement le contact en entraînant par dessous vers L le point du plan qui était joint au cercle. Cependant il revient au même que le plan CL se meuve vers L quand le cercle ne change pas de lieu, ou que le plan demeurant immobile, le cercle soit porté vers $\mathrm{L}^{5}$. Donc un mouvement aussi petit soit-il s'ajoutant au circulaire, ce point du plan auquel se faisait le contact ne peut être joint au plan, sinon dans l'instant; et par conséquent plusieurs points du cercle ne peuvent passer par ce point. C'est pourquoi ni quand le petit cercle parcourt une ligne plus grande que lui, ni quand le grand cercle parcourt une ligne plus petite que lui, il ne se produit un rasement. Voilà la démonstration complète de ce théorème vraiment étonnant, d'une manière qui découvre la cause de tout le mystère, puisqu'elle est en chaque membre ostensive et directe.

Il ne reste qu'un point qu'on voudra peut-être voir expliquer : comment peut-il se faire qu'une circonférence tournée une seule fois parcoure en la touchant une ligne plus grande ou plus petite qu'elle-même ? Il semble en effet qu'on aboutisse par là à égaler en mesure ou en étendue une circonférence à une quantité plus grande ou plus petite, et que par conséquent on égale la plus petite à la plus grande et la plus grande à la plus petite : choses manifestement impossibles. Mais je réponds que, quoique toute la circonférence ait successivement touché toute la ligne du plan, plus grande ou plus petite, pourtant elle ne lui est pas pour autant égalée en mesure ni en étendue; car pour qu'il y ait commensuration successive d'un tout avec un tout, il faut que les parties soient appliquées successivement aux parties et coïncident avec elles. Mais ici aucune partie de la circonférence n'a été appliquée à une partie de la ligne parcourue dans le plan ; mais il y a eu un contact continu indivisible, ou sans quantité. Si un polygone quelconque, un 
hexagone par exemple, tourne sur un plan, son périmètre égale en dimension la ligne faite dans le plan, parce que les parties du périmètre (c'est-à-dire les côtés) ont été appliquées de manière à correspondre. Mais dans le roulement des cercles il en va tout autrement, dans la mesure où la conjonction successive de la circonférence avec la ligne parcourue est toujours indivisible, c'est-à-dire qu'elle reste sans quantité.

\section{Scolie}

\section{Difficultés et mystères du roulement des cercles}

9 Quoique importants et nombreux, à ma connaissance ils n'ont pas pour la plupart été tirés au clair, ni même signalés par personne jusqu’à présent.

1. Le premier au moins a été résolu dans le théorème ci-dessus et sera encore plus pleinement éclairci dans les suivants : comment peut-il assurément se faire que le plus grand s'égale en mesure au plus petit et le plus petit au plus grand, ou mieux que toutes les circonférences inégales possibles s'égalent en quelque manière à la même ligne droite? Et ce qui certes accroît considérablement le caractère étonnant du problème, c'est que celle-ci peut être quelconque; en effet si n'importe quel autre concentrique différent du cercle $A Q$ roule sur un plan matériel, il parcourra en un roulement une droite égale à sa propre circonférence, lui et tous les autres.

2. Mais un problème beaucoup plus difficile encore en découle : pourquoi celui qui tourne sur le plan matériel est-il le seul à parcourir un espace égal à sa circonférence, alors que tous les autres concentriques parcourent des lignes plus grandes ou plus petites que leur périphérie ? Il serait futile de dire que celui qui touche le plan matériel parcourt une ligne égale à lui-même parce qu'il se meut par lui-même et indépendamment des autres, puisqu'on demande ce que c'est qu'être mû par soi et pourquoi celui-ci se meut par soi plutôt que les autres.

3. Qu'arrivera-t-il si aucun des concentriques qui tournent solidairement ne parcourt un plan matériel? On a bien démontré plus haut ${ }^{\mathrm{f}}$ qu'en un roulement tous parcourent un espace égal. Mais quelle sera sa dimension? À la périphérie de quel concentrique sera-t-il égal ? Y en a-t-il alors un qu'on pourra nommer déférent? Dire que les autres sont portés par celui qui est mû en premier et par soi, c'est une réponse insipide, puisque par hypothèse tous les concentriques sont joints et solidaires, de sorte que le mouvement d'un seul les entraîne tous ; il faudrait expliquer ce qui est requis pour que l'un des concentriques se meuve par soi et emporte les autres : cela exposé, il faudra encore montrer que celui qui se meut par soi parcourt un espace égal à sa circonférence.

4. Et si ce cercle qui touche le plan matériel franchit en un roulement un espace égal à sa propre circonférence (personne en tout cas jusqu'ici n'a prouvé autrement que par expérience qu'il en va bien ainsi), qu'arrivera-t-il lorsque deux cylindres EF et CG de même axe $A B$ touchent tous deux des plans matériels parallèles en leurs côtés FE et CG ? Les deux cercles $\mathrm{E}$ et $\mathrm{C}$ parcourront-ils en un roulement des espaces égaux à leurs propres circonférences? C'est pourtant impossible, puisque par la démonstration du premier théorème ci dessus, il est clair que les cercles $\mathrm{E}$ et $\mathrm{C}$ commencent et achèvent leur roulement ensemble, et que par conséquent, tournés tous deux une fois, ils parcourent des espaces égaux. Dirons-nous que dans ce cas le roulement est impossible? Mais on ne voit aucune cause qui l'empêche. Et nous démontrerons ci-dessous qu'il est possible. Quelle sera donc la circonférence égale à l'espace d'un roulement: $\mathrm{E}, \mathrm{C}$ ou aucune des deux ? Si c'est l'une des deux, pourquoi l'une plutôt que l'autre, puisque toutes deux touchent le plan matériel ? Si ce n'est aucune des deux, comme il faut que les cercles $\mathrm{E}$ et $\mathrm{C}$ parcourent tous deux une longueur déterminée, il faudra montrer laquelle, pour répondre à la question. 
5. Les difficultés proposées dans le précédent paragraphe croissent en quelque sorte dans un cône infini, tangent à un plan commun matériel par son côté $\mathrm{FE}$, et emporté sur lui en un roulement droit. Là en effet une infinité de cercles concentriques placés les uns en dehors des autres, touche un plan commun matériel.

10 Ces points et bien d'autres que nous aborderons par la suite montrent assez que le roulement des cercles enferme quelque étonnant mystère de la nature ; jusqu' à présent du moins il n'a pas été éclairci ; cela me semble provenir de ce qu'on n'a pas jeté les yeux sur la proportion des mouvements de translation et circulaire qui composent le roulement, qui peut être diverse et varier à 1 'infini. De fait, la proportion mutuelle de ces deux mouvements correctement évaluée, toutes les difficultés proposées disparaitront, comme les théorèmes suivants le montreront clairement. Faute de cette observation est d'ailleurs née aussi cette commune illusion, que le cercle qui touche un plan matériel parcourt nécessairement, en un roulement complet sur le plan, une ligne égale à sa circonférence, sans qu'il puisse en être autrement. Nous montrerons ci-dessous que c'est faux.

\section{Aristote et Galilée n'éclaircissent rien.}

11 Aristote traite du roulement des cercles concentriques sur un plan dans ses Questions mécaniques, 24. Nous rapportons ici son discours ${ }^{6}$.

12 Si l'on met en mouvement le petit cercle AP, le grand AQ qui lui est attaché ne se meut pas par soi, mais pour autant qu'il est mû par le petit ; par conséquent il effectue avec lui un chemin égal. De même lorsqu'on pousse le grand AQ, alors le petit n'est pas emporté par son propre mouvement, mais par celui du grand: d'où il est nécessaire que réciproquement il accomplisse avec lui en chemin égal. Répondant ensuite à une objection, il dit : quoique les deux cercles n'aient qu'un centre, le centre d'un seul est en mouvement par soi ; l'autre l'est par accident : en effet le cercle qui meut l'autre possède ce centre par soi ; mais l'autre qui est mû l'a par accident, parce qu'il ne s'en sert pas comme de centre.

Voilà le raisonnement d'Aristote dans son ensemble: que cela apporte peu ou pas de lumière à la question, le lecteur le comprendra clairement, s'il compare ce discours d'Aristote aux difficultés proposées dans la précédente section.

Parmi les autres qui ont tenté de défaire ce nœud gordien, le dernier en date, mais le premier en célébrité pour ses nombreuses inventions très connues et subtiles en mathématiques, s'appelle Galilée le Lynx. Dans le premier dialogue de son livre intitulé Discorsi e demonstrazioni matematiche etc il tente d'éclaircir le premier point de notre précédent théorème seulement : comment se peut-il que la petite circonférence parcoure une ligne plus grande que soi, et la grande une plus petite en un roulement commun? Voici comment il raisonne. 


\section{Schematismus XVII}

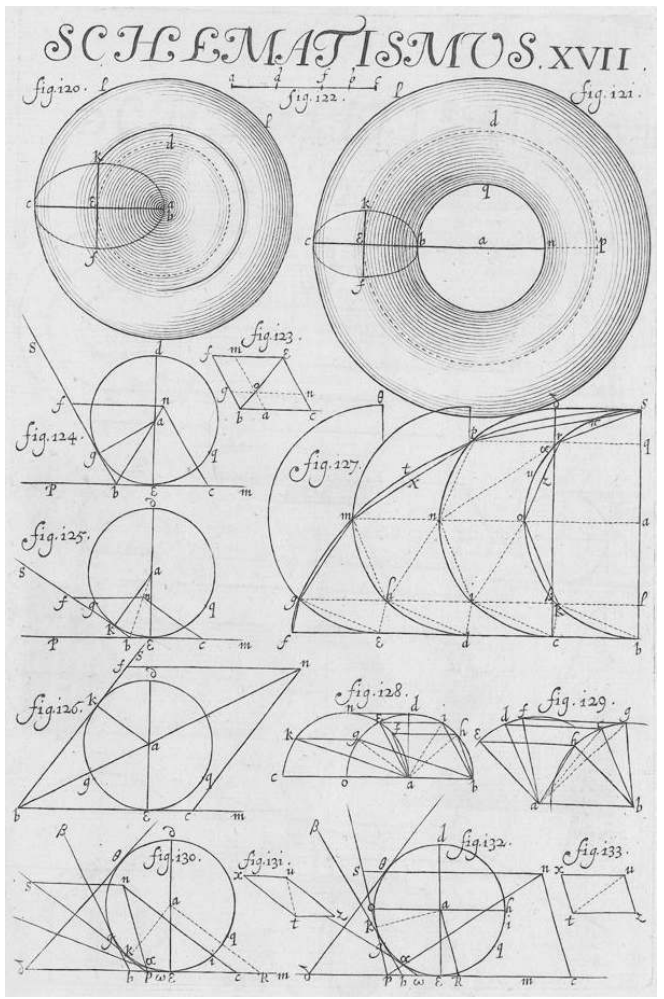

Planche illustrée des figures géométriques.

\section{Schematismus XVIII}

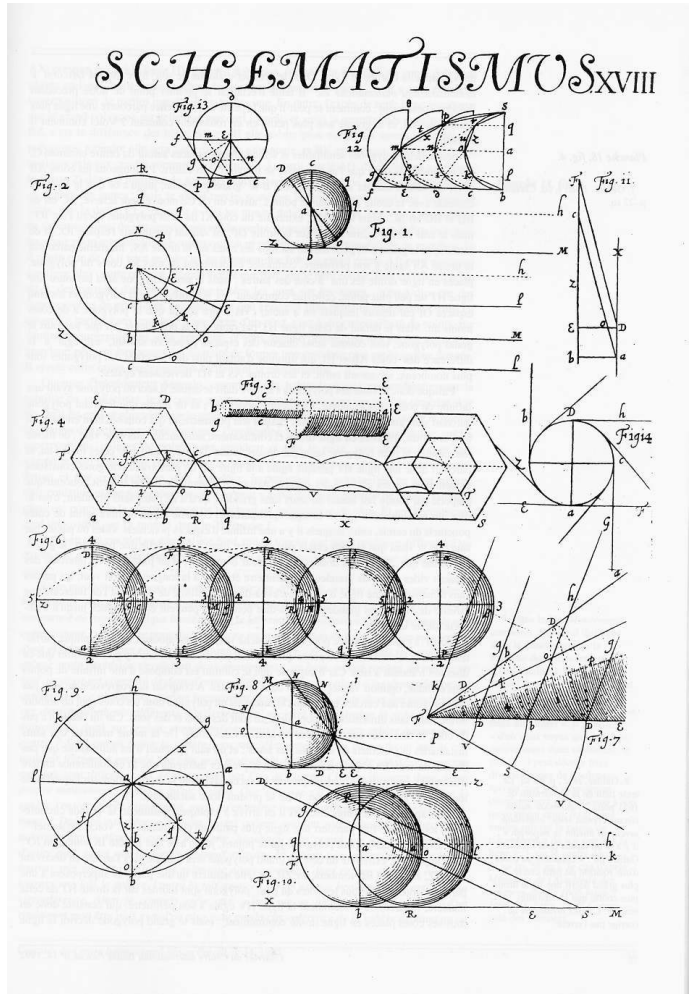

Planche illustrée des figures géométriques. 
Soient deux polygones semblables et semblablement placés autour du centre commun G, liés ensemble de sorte que l'un ne puisse se mouvoir sans l'autre. Prolongeons les côtés $\mathrm{AB}$ et $\mathrm{HI}^{7}$ ). Faisons tourner sur le point fixe $\mathrm{B}$ le grand polygone, jusqu'à ce que le côté $\mathrm{BC}$ coïncide avec la droite $A S$ et que le point $C$ arrive en $Q$. Ce mouvement achevé, $B C$ est en $\mathrm{BQ}$ et $\mathrm{BG}$ en $\mathrm{BC}$. Mais le point I, extrémité du côté $\mathrm{KI}$ du petit polygone, décrit l'arc IO ; mais le côté KI lui-même tombe sur la droite OP, en sautant par-dessus l'espace IO. Si de cette façon le grand polygone tourne sur tous ses côtés sur la droite AS, lui-même parcourra la droite AS égale à son périmètre, composée évidemment de tous les côtés du polygone, placés en ligne droite les uns à côté des autres : mais le petit polygone aura parcouru une ligne HT un peu plus petite, celle que composent les six côtés du petit polygone et les cinq espaces OI par-dessus lesquels on a sauté, c'est-à-dire autant que le polygone a de côtés moins un. Mais le défaut de cette ligne HT par rapport à la précédente AS que parcourt le grand polygone, tout comme aussi chacun des espaces passés en sautant, est égal à la différence des côtés $\mathrm{AB}$ et $\mathrm{HI}$, qui diminue d'autant plus que les côtés des polygones sont plus nombreux, s'évanouit enfin, et les droites AS et HT deviennent égales.

16 Puisque donc la suite des polygones s'achève dans le cercle, il sera un polygone ayant une infinité de côtés sans grandeur, c'est-à-dire ponctuels ; et de même que le grand polygone parcourt sans aucun saut la ligne AS, égale à son périmètre, et qui consiste bien entendu en tous ses côtés, disposés en ligne droite et continûment, sans aucun intervalle vide ; de même tout comme le petit polygone saute par-dessus autant d'espaces qu'il a de côtés moins un, et parcourt donc une ligne HT presque égale à la ligne AS du plus grand polygone, consistant évidemment en ses côtés et des espaces passés en sautant, de même le petit concentrique emporté par l'autre fait autant de sauts sans grandeur qu'il a de côtés sans grandeur, c'est-àdire un nombre infini : aussi la ligne qu'il parcourt consiste en un nombre infini de côtés ponctuels du cercle, entre lesquels il y a une infinité d'espaces ponctuels vides ou passés par saut. De là vient que le petit cercle parcourt une ligne égale à celle que parcourt le grand. De même que les parties douées de grandeur d'une ligne d'un pied, si on y intercale des espaces vides doués de grandeur, peuvent être étendus à la longueur qu'on veut, les parties sans dimension d'une ligne d'un pied, c'est-à-dire une infinité de points, si l'on intercale une infinité de vides sans grandeur, c'est-à-dire ponctuels, peuvent être étendues jusqu'à n'importe quelle longueur.

17 Ainsi s'exprime Galilée pour ce qui touche la question, ajoutant qu'on explique parfaitement ainsi la raréfaction. Je pense qu'il ne faut pas discuter beaucoup pour montrer que ce discours n'aboutit à rien. Car il suppose que le continu est composé d'une infinité de points sans étendue, opinion rejetée partout dans les écoles. À coup sûr la progression ne vaut pas des polygones aux cercles, et dire que le cercle est un polygone dont les côtés sont en nombre infini, mais sans dimension ou ponctuels, ce sont des mots et des sons. Car un cercle n'a pas de côté, et des côtés sans grandeur ne sont pas des côtés. De la même manière, des sauts instantanés et ponctuels ne sont pas des sauts; et un saut ponctuel n'est autre chose que pas de saut du tout. En plus on a montré dans le premier théorème que la circonférence entière d'un cercle concentrique quelconque touche successivement la droite entière sur laquelle elle tourne sans aucune interpolation. Il ne se produit donc aucun saut.

18 Puis après bien de longs discours il en arrive à expliquer comment la grande circonférence tournée une fois parcourt une ligne plus petite qu'elle-même. En voici l'essentiel $^{8}$. Faisons tourner le petit polygone sur le point I, jusqu'à ce que le côté IK 
tombe en IO : alors le point $\mathrm{B}$, extrémité du côté du grand polygone sera ramené vers l'arrière en décrivant l'arc $\mathrm{BZ}$, et le côté $\mathrm{BC}$ tombera sur $\mathrm{ZR}$, de telle manière qu'une partie se superposera à une partie du côté $\mathrm{AB}$. Si tous les côtés du petit polygone sont tournés sur la droite HT de cette manière, il parcourra évidemment la ligne HV égale à son périmètre, qui consiste donc en tous ses côtés placés en ligne droite continûment: mais le grand polygone décrira la ligne $\mathrm{AX}$, presque égale à la ligne $\mathrm{HV}$ du petit polygone, formée par les côtés du grand polygone placés en ligne droite, mais tombant les uns sur les autres en partie ; et il y aura autant de superpositions de parties que de côtés, moins une. En outre, l'excès de cette ligne parcourue par le grand polygone sur celle que parcourt le petit, et aussi la quantité des superpositions, BZ, c'est la différence des côtés $\mathrm{AB}$ et HI elle-même plus nombreux seront les côtés des polygones, moindre elle sera ; elle finit par s'évanouir, et $\mathrm{HV}$ et AX par devenir égales. Il applique cela aux cercles comme précédemment. Le petit cercle AQ, dit-il, mû par soi (voyez la figure 2) déroule l'infinité de ses côtés sans grandeur sur la droite BM, tous posés les uns en dehors des autres : mais le grand cercle, porté par le petit, dépose aussi son infinité de côtés sur la droite CL, pas tout à fait les uns en dehors des autres, mais avec une infinité de superpositions, de rétrogradations ou de contractions sans grandeur, c'est-à-dire ponctuelles, mais pourtant sans aucune superposition ni pénétration de parties douées de grandeur. Et il pense que de là il peut se faire qu'en tournant une fois, le grand cercle AR peut parcourir une ligne plus courte que soi et égale à celle que parcourt le petit cercle. Tout comme en effet, ditil, des parties d'un pied de long posées les unes sur les autres, c'est-à-dire par des superpositions de grandeurs, peuvent faire une grandeur plus petite qu'une donnée, ainsi en retour les parties sans grandeur d'une ligne d'un pied, c'est-à-dire une infinité de points, par d'infinies superpositions sans grandeur, et sans aucune pénétration des parties douées de grandeur, peuvent être resserrées au point de former une grandeur plus petite qu'une donnée. Il ajoute enfin qu'on explique ainsi facilement la condensation. Quoique l'interlocuteur Sagredo, comme à son habitude, vante extraordinairement la subtilité de ce discours, je ne crains guère que le lecteur savant ne me taxe de témérité si je dis qu'il est encore plus vide que le précédent. Car bien qu'il explique avec justesse et finesse la rotation des polygones concentriques, l'application aux cercles, tout ce qu'il ajoute sur les superpositions infinies de points sans grandeur et tout le reste, sont de ces choses que n'importe quel philosophe peut réfuter facilement. Je ne réponds que d'un mot : une rétrogradation et une superposition sans grandeur, instantanées ou ponctuelles ne sont ni une rétrogradation ni une superposition; et il ne peut y avoir une superposition de points, sans que plusieurs points de la circonférence ne touchent un même point du plan. Et en l'occurrence c'est impossible, on l'a montré dans le Théorème 1.

20 Voilà en tout cas tout ce que Galilée écrit, qui ne touche que notre première difficulté. Des autres que nous nous sommes proposées, ni lui ni un autre ne les mentionne, à ma connaissance.

Reste maintenant, si nous pouvons éclaircir ces difficultés, à nous exécuter par ordre et brièvement. Avant de le faire, il ne sera pas hors de propos de rappeler par quelle voie j'ai commencé de trouver quelque lumière dans le labyrinthe. Je voyais ce que tout le monde voit certainement : une sphère poussée du doigt se meut sur un plan en avançant d'un mouvement droit et en même temps en tournant autour de son centre ; et je remarquais par conséquent qu'une seule impulsion engendrait deux mouvements, ou bien, ce qui revient au même, un seul mouvement composé de deux d'espèce différente ${ }^{9}$. $\mathrm{J}$ e vins alors à me demander ce qui arriverait si ce n'était pas, comme auparavant, un seul moteur, mais deux qui produisaient ces 
deux mouvements différents. Qu'arriverait-il en effet l'un entraîne la sphère dans un mouvement droit sur le plan, et qu'en même temps l'autre tourne cette sphère autour de son centre ? Sans aucun doute la sphère sera mue exactement de la même façon que si elle tournait librement poussée par un seul moteur. Je raisonnais ensuite ainsi : comme ces moteurs sont différents, ils ne dépendent pas l'un de l'autre. Chacun donnera donc à son propre mouvement la vitesse qu'on voudra. Que se passe-t-il donc si celui qui entraîne la sphère l'entraîne très rapidement, mais qu'en même temps l'autre la fait tourner très lentement? Dans ce cas nécessairement, avant qu'une rotation soit achevée, la sphère aura parcouru sur le plan un espace très long, beaucoup plus grand que sa circonférence. Si d'autre part, alors que le premier moteur entraîne la sphère très lentement, l'autre la fait tourner très vite, $\mathrm{j}$ 'ai vu tout de suite que nécessairement, dans ce peu de temps où il fait tourner une fois la sphère, l'autre la fait avancer sur un espace beaucoup plus petit que sa circonférence. Ainsi je compris enfin que, même sur un plan matériel, le chemin d'un roulement peut être plus grand ou plus petit que la circonférence de la sphère d'autant qu'on le veut; et par suite toute l'affaire dépend, non pas de la vitesse ou de la lenteur de chacun des mouvements pris séparément, mais des deux comparés l'un à l'autre et de leur proportion mutuelle.

Ces préalables posés, je viens à mon sujet; nous envisagerons dans les premiers théorèmes les roulements sur des plans géométriques. Nous étudierons ensuite le régime des roulements sur un plan matériel commun, et dans le plan matériel parfait. Enfin nous examinerons la ligne courbe même que décrit chaque point $d u$ cercle lorsqu'il roule, et quelques autres aspects des roulements.

\section{Théorème II}

21 Si le mouvement parallèle au plan par lequel est porté le centre d'un cercle c'est-àdire le cercle même, et le mouvement circulaire par lequel tourne autour de son centre sont d'égale vitesse, la ligne que le cercle parcourt en un roulement sur le plan est égale à la circonférence.

\section{parcourra en un roulement une ligne plus grande que sa circonférence ; mais il en parcourra une plus petite si le mouvement parallèle est plus lent que le circulaire.}

Puisque par hypothèse le mouvement de translation et le mouvement circulaire sont de même vitesse, par l'axiome 1 ils parcourent des espaces égaux. L'espace que parcourt le centre par le mouvement de translation dans le temps d'un roulement est égal à la ligne que le cercle parcourt dans le plan en une rotation en le touchant continûment; mais l'espace qu'accomplit le mouvement circulaire dans le temps d'un roulement est la circonférence même, comme il est clair par la définition 5. Donc la ligne droite qu'en un roulement le cercle parcourt dans le plan qu'il touche continûment est égale à la circonférence. Ce qu'il fallait démontrer.

La démonstration de la seconde partie se déduira du second théorème universel.

\section{Corollaire}

Si la droite parcourue en un roulement est égale à la circonférence, le mouvement parallèle du centre et le circulaire seront d'égale vitesse. 


\section{Théorème III}

Universellement, le rapport de la vitesse du mouvement parallèle qui porte le centre à la vitesse du mouvement circulaire est le même que celui de la ligne que le cercle parcourt en un roulement sur le plan à la circonférence de ce cercle.

L'espace parcouru dans le temps d'un roulement par le mouvement parallèle du centre est égal à la ligne que le cercle parcourt tangentiellement sur le plan en un roulement. Mais l'espace qu'accomplit le mouvement circulaire pendant un roulement est la circonférence même; car par la définition 5 en un roulement elle tourne entièrement une fois. Et selon l'axiome 2, Galilée a démontré dans son deuxième théorème sur le mouvement uniforme que le rapport des vitesses est identique à celui des espaces parcourus dans le même temps; donc dans le cas donné aussi le rapport de la vitesse du mouvement de translation à celle du mouvement circulaire est le même que celui de l'espace accompli pendant un roulement par le mouvement de translation à l'espace accompli dans le même temps d'un roulement par le mouvement circulaire. Donc le rapport de la vitesse du mouvement de translation ou parallèle à la vitesse du mouvement circulaire est identique à celui de la ligne droite que le cercle tangent parcourt en un roulement sur le plan à la circonférence même du cercle. Ce qu'il fallait démontrer.

\section{Théorème IV}

De cela on pourra facilement déduire, étant donné un roulement quelconque, parmi une infinité de cercles concentriques sur des plans géométriques, lequel est le seul qui accomplit en un roulement une ligne égale à sa circonférence.

Ce sera en fait celui qui tourne autour du centre avec une vitesse égale à celle qui emporte le centre dans le mouvement parallèle au plan. On l'appellera à juste titre primaire ou déférent.

Mais je démontre comme suit que, pour un roulement quelconque donné, on trouvera toujours un cercle et un seul qui tourne autour du centre avec la même vitesse que le centre avance dans le mouvement parallèle. Puisqu'on a montré dans le Théorème 1 que tous les concentriques parcourent en un roulement des lignes droites égales, autrement dit la même, posons que ce soit BM (voir figure 2). Il est possible de décrire autour du centre $\mathrm{A}$ une circonférence égale à la droite BM. Posons que ce soit $\mathrm{Q}$. Donc puisque tous les concentriques parcourent en un roulement des lignes égales à $\mathrm{BM}$, en un roulement le concentrique $\mathrm{AQ}$ aussi parcourra $\mathrm{BM}$, égale à sa propre circonférence. Donc par le corollaire du Théorème II, le concentrique $\mathrm{AQ}$ tourne autour du centre $\mathrm{A}$ avec une vitesse égale à celle du centre A dans son mouvement parallèle au plan; d'où il apparaît aussi qu'il n'y a qu'un seul cercle de ce genre.

\section{Théorème $\mathrm{V}$}

31 C'est pourquoi les concentriques plus petits que le primaire parcourent en un roulement des lignes plus grandes que leur circonférence; en revanche les concentriques plus grands que le primaire accomplissent des droites plus petites que leur circonférence. 
Soit $\mathrm{AQ}$ le cercle primaire qui parcourt une ligne égale à sa circonférence ; et soit pris un concentrique quelconque plus petit AN. Puisque tous les cercles tournent ensemble autour du centre commun A, il est évident que le petit AN tourne plus lentement que le grand $\mathrm{AQ}$. Mais comme par hypothèse $\mathrm{AQ}$ parcourt une ligne égale à sa circonférence, la rotation ou le mouvement circulaire du cercle $A Q$ est aussi rapide, par le Théorème II, que le mouvement de progression du centre commun A. Donc le mouvement circulaire du cercle AN est plus lent que le mouvement de progression du centre. Donc par le Théorème III le cercle AN en un roulement parcourt une ligne plus grande que sa circonférence.

Prenons ensuite un concentrique quelconque AR plus grand que AQ. Il est clair que le plus grand AR tourne plus vite que $A Q$ le petit. C'est pourquoi, puisque le mouvement circulaire de $\mathrm{AQ}$ est aussi rapide que le mouvement parallèle du centre commun $\mathrm{A}$, le mouvement circulaire de AR est plus rapide que le mouvement parallèle du centre commun A. Donc par le Théorème III le cercle AR en un roulement parcourt un espace plus petit que sa circonférence. On connaît donc la raison pour laquelle etc.

\section{Théorème VI}

\section{D'où vient que l'infinité des concentriques inégaux parcourt en un roulement une ligne égale?}

Soit $\mathrm{AQ}$ le concentrique qui parcourt la ligne $\mathrm{BM}$ égale à sa circonférence ; et prenons en un autre quelconque AP, plus grand ou plus petit. Puisque par hypothèse tous les concentriques accomplissent dans le même temps une rotation complète autour du centre commun $\mathrm{A}$, par l'axiome 2 le rapport de la circonférence $\mathrm{Q}$ à la circonférence $\mathrm{P}$ sera le même que celui de la vitesse avec laquelle tourne $Q$ à la vitesse avec laquelle tourne $\mathrm{P}$. Cependant la droite BM est par hypothèse égale à la circonférence $\mathrm{Q}$, et la vitesse du mouvement parallèle qui emporte le centre A dans le roulement est égale par le corollaire du Théorème II à la vitesse avec laquelle la circonférence $Q$ tourne autour $d u$ centre $\mathrm{A}$, puisque par hypothèse la droite $\mathrm{BM}$, qui est parcourue par le cercle $\mathrm{AQ}$ en un roulement, est égale à la circonférence $\mathrm{Q}$. Donc Je rapport de la droite BM à la circonférence $\mathrm{P}$ est Je même que celui de la vitesse du mouvement parallèle qui emporte le centre commun A à la vitesse avec laquelle tourne la circonférence P. Donc par le Théorème III la droite $\mathrm{BM}$ est celle que parcourt le concentrique quelconque AP en un roulement. On connaît donc la raison pour laquelle l'infinité des concentriques parcourt la même ligne en un roulement.

Figure 2

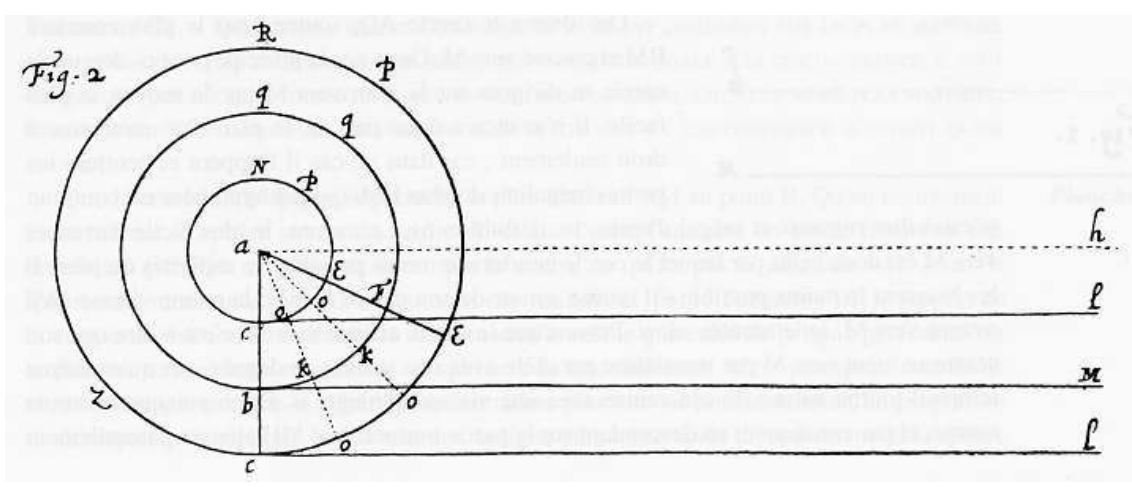




\section{Théorème VII}

D'où vient que la ligne que l'infinité des concentriques mus ensemble parcourt en un roulement peut être tantôt plus grande, tantôt plus petite, ou plutôt quelconque?

À coup sûr le fait que le centre commun soit porté ou les cercles tournés autour du centre commun plus ou moins vite ne fait rien à l'affaire; mais tout dépend de la proportion relative de leurs mouvements.

Soient donc donnés en nombre quelconque des cercles autour du centre commun A, ainsi qu'une ligne quelconque BM. On peut tracer autour du centre A une circonférence égale à la droite donnée BM. Que ce soit Q. De nouveau on peut faire tourner les concentriques de sorte que le mouvement circulaire du cercle AQ autour du centre commun A soit aussi rapide que le mouvement parallèle qui porte le centre commun A. Mais alors le concentrique $\mathrm{AQ}$, par le Théorème II, parcourra en un roulement une droite égale à sa circonférence $Q$, c'est-à-dire par construction la ligne BM. Mais tous les concentriques parcourent des lignes égales, comme on l'a montré aux Théorèmes I et VI. Donc tous les concentriques parcourent en un roulement des lignes égales à la ligne donnée BM. On connaît donc la raison pour laquelle etc.

\section{Corollaire}

Toutes les démonstrations précédentes montrent manifestement que la grandeur de la ligne parcourue en un roulement dépend seulement de la proportion des deux mouvements dont est composé le roulement, savoir les mouvements de translation et circulaire.

40 Nous avons à présent assez considéré les roulements dans le cas général, savoir ceux qui se font sur les plans géométriques, abstraits de toute matière; passons maintenant aux plans matériels.

\section{Théorème VIII}

41 D'où vient que lorsque la sphère ou le cercle tournent librement sur un plan commun matériel, ils parcourent une ligne égale à leur circonférence.

42 Je ne vois personne qui montre, autrement que par expérience, qu'il en va bien ainsi, et qui en dévoile la raison. Il est évident par les démonstrations précédentes qu'un cercle quelconque, lorsqu'il roule sur un plan géométrique, parcourt en un roulement un espace qui peut être plus grand ou plus petit que sa circonférence d'autant qu'on le veut. Mais que produit le contact d'un plan matériel, pour engendrer cette égalité constante entre la circonférence du cercle et l'espace parcouru en un roulement? En vue de cette recherche, posons ce principe :

43 La Nature, ne faisant rien en vain, tout comme elle agit par la voie la plus brève, prend aussi le moyen le plus facile. 
Planche 18, Figure 1

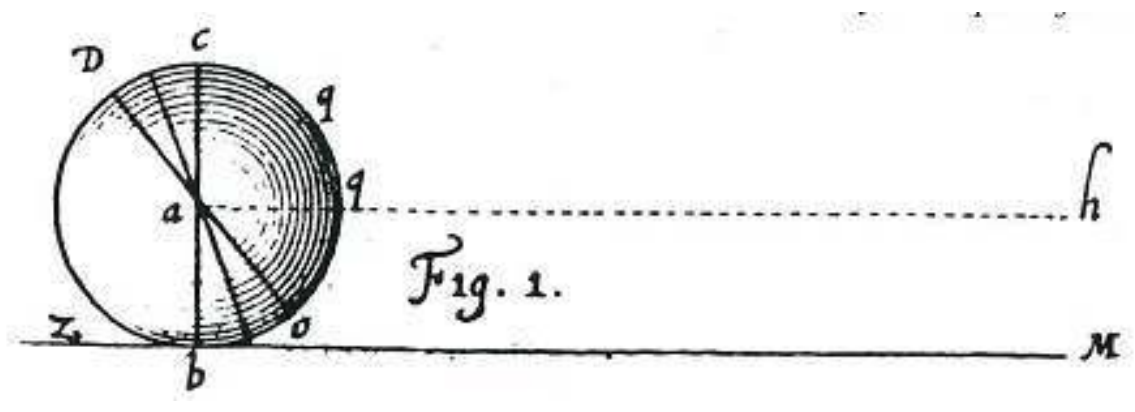

On donne le cercle $\mathrm{AQ}$, soutenu par le plan commun BM et poussé vers $\mathrm{M}$. Donc par le principe posé ci-dessus, le cercle se dirigera sur le plan vers $\mathrm{M}$ par le moyen le plus facile. Il n'avancera donc pas sur le plan d'un mouvement droit seulement; car dans ce cas il frappera et heurtera les petites inégalités du plan BM, qui par hypothèse est commun (c'est-à-dire rugueux et inégal d'après la définition 6). Le moyen le plus facile d'avancer vers $M$ est donc celui par lequel le cercle heurtera le moins possible les aspérités du plan. Il les heurtera le moins possible s'il tourne autour de son centre A avec la même vitesse qu'il avance vers $\mathrm{M}$. Je le montre ainsi. Posons que le cercle avance vers $\mathrm{M}$, c'est-à-dire que son centre se meut vers $M$ par translation parallèle avec une vitesse de degré 6 ; et qu'en même temps il tourne autour de son centre avec une vitesse de degré 2. Donc puisque le cercle tourne, et par conséquent en descendant sur la partie tournée vers $\mathrm{MH}$ abaisse naturellement sa circonférence sur le plan, mais en montant avec l'autre partie soulève cette même circonférence au-dessus du plan, il est manifeste qu'il frappe et heurte déjà moins les petits reliefs du plan. Pourtant parce que la translation vers $\mathrm{MH}$, qui tant qu'il n'y avait aucun mouvement circulaire, était la cause propre du heurt, est plus rapide que le mouvement circulaire, c'est-à-dire que l'abaissement et l'élévation indépendants de la circonférence, il ne reste plus d'empêchement qu'autant que la vitesse de translation vers MH surpasse la vitesse de la rotation. C'est pourquoi si la vitesse de la rotation du cercle autour du centre devient la même absolument que celle de la translation vers $\mathrm{MH}$, alors tout empêchement à la progression est supprimé. Car tourner le cercle avec la même vitesse qu'il progresse ou se porte vers $M$, c'est précisément abaisser successivement de son propre mouvement toutes les parties de la circonférence sur le plan et les soulever de l'autre côté avec cette même vitesse avec laquelle se fait la progression vers M. Mais alors aucune partie de la circonférence ne peut heurter le plan ; car comme la rotation et la progression sont d'égale vitesse, les parties de la circonférence sont sou levées du plan à l'instant même où elles devraient le heurter.

$\mathrm{Si}$ la rotation devient plus rapide que le mouvement de translation vers $\mathrm{MH}$, je montre ainsi que de nouveau le cercle heurte le plan. Concevons qu'un cercle posé sur un plan commun tourne autour du centre A dans le sens des lettres CQBD, sans aucun mouvement de translation vers $\mathrm{MH}$. Il est évident que le cercle heurtera continuellement les petites aspérités du plan placées autour du contact, tout comme il les heurtait auparavant, lorsqu'il était porté vers MH sans rotation autour du centre A. Si ensuite au mouvement circulaire s'ajoute un mouvement de translation vers $\mathrm{MH}$, quoique moins rapide encore que le circulaire, il n'en fera pas moins que le cercle en tendant vers $\mathrm{MH}$, soit un peu emmené, et par conséquent heurte déjà moins que lorsqu'il restait au même endroit, en tournant seulement autour du centre A. Pourtant même alors il heurtera encore, parce que le mouvement de rotation qui était la cause propre du choc alors qu'il n'y avait pas de 
translation vers $\mathrm{MH}$, est plus rapide encore que le mouvement de translation vers $\mathrm{MH}$ : de là vient que les parties du cercle descendent sur les aspérités du plan plus vite qu'elles n'en sont emmenées par le mouvement de translation. Si le mouvement de translation ou la progression du centre vers MH devient aussi rapide que le mouvement de rotation, il apparait évidemment que la circonférence descend sur les aspérités du plan par le mouvement circulaire avec la même vitesse qu'elle en est soulevée par le mouvement de translation. C'est pourquoi tout choc cesse alors de nouveau. Ce n'est donc pas seulement un mouvement circulaire plus lent que le mouvement de translation qui cause le heurt, mais aussi sa plus grande rapidité ; mais lorsqu'ils sont égaux tout choc cesse.

C'est pourquoi la manière la plus facile correspond au cas où la rotation du cercle et la progression du centre sont de même vitesse. Donc par le principe posé plus haut, le cercle parcourt le plan vers $\mathrm{M}$ de cette façon. Cependant par le second Théorème, lorsque la rotation ou le mouvement circulaire et la progression du centre ont la même vitesse, le cercle parcourt en un roulement une ligne égale à sa circonférence. Donc lorsque le cercle tourne librement sur un plan matériel commun, il accomplit en un roulement une ligne égale à sa circonférence. Nous avons donc démontré la proposition, pour le fait et pour la cause.

\section{Théorème IX}

Quoique la sphère ou le cercle roulant librement sur un plan matériel commun accomplisse toujours en un roulement une ligne égale à sa circonférence, il peut cependant arriver que, tourné une fois, en touchant successivement le même plan matériel, il parcourt une ligne plus grande ou plus petite que sa circonférence d'autant qu'on voudra.

Que le cercle ou la sphère $A Q$ touche le plan matériel $B M$ au point $B$. Qu'un mouvement fasse tourner la sphère autour du centre $A$, et que l'autre l'entraîne en même temps en une translation parallèle au plan, de sorte que le centre ne quitte pas la parallèle AH. Alors la sphère sera mue sur le plan BM, comme si elle roulait : et par le Théorème III, le rapport de la vitesse de traction à la vitesse de rotation sera le même que celui de la ligne parcourue sur le plan au terme d'une rotation de la sphère à la circonférence. C'est pourquoi, comme le mouvement qui entraîne la sphère peut l'entraîner plus vite ou plus lentement, en proportion quelconque, que celui qui la fait tourner, la ligne parcourue après un roulement de la sphère pourra être plus grande ou plus petite que la circonférence, en proportion quelconque. Ce qu'il fallait démontrer.

\section{Théorème $X$}

\section{Étude du roulement rectiligne d'un cône sur un plan géométrique.}

Soit un cône infini (c'est-à-dire indéfini) qui touche un plan géométrique par son côté FE. Coupons ensuite ce cône par une infinité de plans qui y engendrent une infinité de cercles D, B, etc., et dans le plan tangent au cône, des droites DG, BH. Roulons ensuite le cône sur le plan d'un roulement droit, c'est-à-dire de telle sorte que le cercle D ne quitte pas la droite $D G$; ceci posé, tous les cercles qui restent ne quitteront pas non plus les tangentes $\mathrm{BH}$, etc. Avec ces données, je dis que :

1. Tous les cercles commencent et achèvent leur roulement en même temps : tous en effet touchent le plan aux points du même côté EF. 
2. De cette infinité de cercles un seul parcourt en un roulement une longueur égale à sa circonférence, savoir celui qui tourne autour de l'axe commun FP avec la même vitesse que l'axe lui-même avance d'un mouvement droit.

3. Les autres cercles en nombre infini parcourent des lignes égales à celui-là.

4. Cette longueur commune, que tous les cercles parcourent en un roulement, peut être quelconque.

51 De tout cela les démonstrations et les raisons se déduisent des sept premiers théorèmes.

\section{Théorème XI} seul sur la ligne $\mathrm{BH}$, il tournerait autour du centre ou de l'axe FP avec la même vitesse qu'il avance vers $\mathrm{H}$ : car c'est le moyen le plus facile pour le cercle de se mouvoir vers $\mathrm{H}$, comme le démontre le Théorème VIII. Mais puisque le cercle B ne peut tourner sans que, par hypothèse, tous les autres tournent aussi, si la rotation du cercle B est aussi rapide que le mouvement droit qui emporte le centre, la rotation des autres sera plus rapide ou plus lente que le mouvement droit du centre du cercle B; et plus rapide ou plus lente selon que le circonférences des autres cercles sont supérieures ou inférieures à la circonférence de $\mathrm{B}$, comme le montre l'axiome 2. Donc tous les cercles (c'est-à-dire la surface du cône entière), sauf le seul cercle $B$, frapperont les petites inégalités du plan commun, comme nous l'avons prouvé dans la démonstration du Théorème VIII. On connaît donc la raison pour laquelle etc. 


\section{Corollaire}

58 On appliquera facilement ce qui vient d'être dit du cône aux cylindres de même axe et de largeur différente.

\section{Théorème XIII}

D'où vient qu'un cône peut très facilement rouler circulairement par soi-même autour de son centre sur un plan commun.

En voici la raison. D'après le principe posé au Théorème VIII, la Nature agit toujours par le moyen le plus facile. L'impulsion imprimée au cône par la poussée le portera de façon qu'il franchisse le plus facilement l'aspérité d'un plan commun. Mais la démonstration du Théorème VII prouve qu'un cercle franchit le plus facilement l'aspérité d'un plan commun lorsqu'il tourne avec la même vitesse que le centre avance parallèlement au plan. Donc le cône aussi, qui est une espèce d'amas de cercles décroissants en nombre infini franchira le plus facilement l'aspérité des plans communs lorsque tous les cercles concevables tourneront également avec la même vitesse que tous les centres dans le mouvement parallèle au plan. Donc seul ce genre d'impulsion pourra mouvoir le cône d'une façon qui rendra les mouvements circulaires de tous égaux en vitesse aux mouvements parallèles des centres. Mais que ce soit le roulement circulaire et lui seul, je le démontre ainsi.

61 Le sommet $\mathrm{F}$ restant fixe, que le cône tourne circulairement, de façon que le côté $\mathrm{FO}$, qui au début se trouvait en haut, se retrouve sur FVS dans le plan. Supposons qu'un des cercles du cône, B par exemple, tourne avec une vitesse égale à celle du centre dans son mouvement parallèle au plan. Par le Théorème II l'arc B4Q sera donc égal à la partie de la circonférence BS. Et le rapport de l'arc B4Q à l'arc DIO est le même que celui de la périphérie entière $B$ à la périphérie entière $D$; c'est-à-dire du diamètre $B B$ au diamètre DD ; c'est-à-dire de FB à FD ; et pareillement le rapport de l'arc BS à l'arc DV est comme celui de FB à FD. Donc le rapport de l'arc B4Q à l'arc DIO est comme celui de l'arc BS à l'arc DV. C'est pourquoi comme les arcs B4Q et BS sont égaux, les arcs DIO et DV sont égaux aussi. Le centre du cercle $\mathrm{D}$ parcourt donc dans son mouvement parallèle une ligne égale à l'arc DV, dans le temps que tourne la partie de circonférence DIO. Donc par le corollaire du Théorème Il, le mouvement parallèle qui fait avancer le centre du cercle $\mathrm{D}$, et le mouvement circulaire du cercle $\mathrm{D}$ autour du centre, sont de vitesse égale. Une démonstration semblable vaut pour tous les autres. C'est pourquoi, si un cône roule circulairement, tous les mouvements circulaires des cercles possibles dans le cône égalent en vitesse les mouvements parallèles des centres. Que cela ne puisse être l'effet que du seul roulement circulaire autour du sommet, on le conclut facilement des précédentes démonstrations.

On connaît donc la raison pour laquelle un cône poussé par un moteur, lorsqu'il est laissé à lui-même et que le sommet reste fixe, tourne circulairement ; il apparaît du même coup d'où vient que, dans chacun de ces roulements orbiculaires, les circonférences de chacun des cercles du cône parcourent dans le plan commun des arcs qui leur sont égaux, et que la surface du cône entier parcourt dans le plan un secteur qui lui est égal.

Une sphère qui roule naturellement sur un plan commun matériel parcourt une ligne égale à la circonférence du grand cercle, comme il a été montré au Théorème VIII. Mais il en va tout 
autrement lorsqu'elle roule sur un plan matériel parfait; pour étudier ce point, nous ajoutons les cinq théorèmes suivants. Si le lecteur savant y trouve quelque sujet de désaccord avec nous, il doit comprendre que je ne les propose pas avec la même certitude que les précédents: ce ne sont pas toujours des démonstrations, mais mes réflexions physicomathématiques, qu'à l'exemple des grands hommes j'ai voulu consigner par écrit. Note: Dans ce qui suit, par plan matériel parfait, j'entends une partie d'une surface sphérique parfaite, ayant pour centre le centre de la Terre.

\section{Théorème XIV}

Si une sphère soutenue par un plan matériel parfait est poussée selon une ligne parallèle au plan, elle sera portée par le seul mouvement de translation, sans nulle rotation autour de son centre.

Figure 10

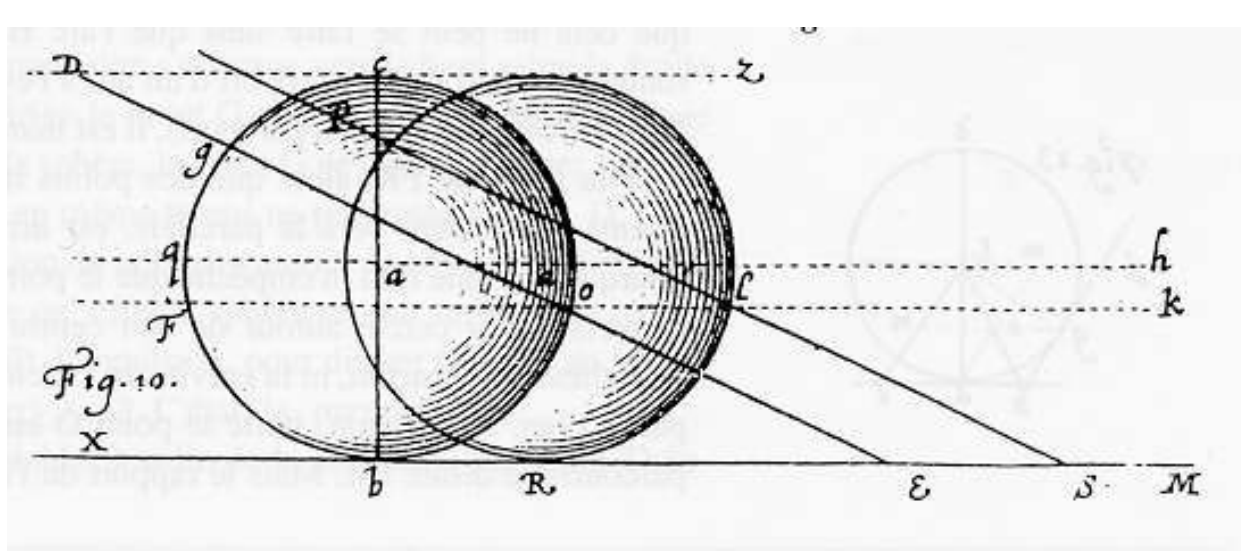

Que le moteur pousse selon la droite FO parallèle au plan XM, qu'elle passe ou non par le centre A. Puisque la sphère est entièrement soutenue par le plan, et que l'impulsion selon la parallèle FO laisse entière cette sustentation parfaite, il est évident que la sphère ne peut en aucune manière résister à une telle impulsion. Mais le plan non plus ne résiste pas à l'impulsion, puisque par hypothèse d'une part le plan lui-même est dépourvu de toute inégalité, et d'autre part l'impulsion, étant parallèle, n'est pas opposée au plan. Donc le point 0 , vers lequel l'impulsion est d'abord dirigée, sera porté suivant la parallèle d'impulsion. La proposition est par là évidente.

\section{Théorème XV}

Si un cercle, ou une sphère, soutenu en B par un plan matériel parfait est poussé obliquement vers le bas suivant une droite $\mathrm{DOE}$, qui coupe le plan en $\mathrm{E}$, la sphère roulera, et l'espace qu'elle accomplira en un roulement aura la circonférence de la sphère de la même raison que la ligne BE à l'arc BO.

Démonstration de la deuxième partie. Puisque l'impulsion se fait vers le bas suivant la droite DOE, le mouvement va de manière à diriger le point 0 vers $E$. Mais la solidité du plan empêche le point 0 de se diriger en descendant vers $E$ sans que la sphère ne tourne autour du centre $\mathrm{A}$, et du même coup n'avance vers $\mathrm{M}$; et rien n'empêche la rotation de la sphère autour de son centre, ni sa translation vers $M$, puisque d'une part le plan est 
dénué de toute aspérité, et que d'autre part le soutien parfait du plan supprime la gravité de la sphère. La poussée imprimée par l'impulsion pour diriger 0 en $\mathrm{E}$ fait donc avancer la sphère vers $\mathrm{MH}$ en la faisant tourner autour du centre $\mathrm{A}$, c'est-à-dire que la sphère roule. C'était le premier point.

Démonstration de la deuxième partie. Soit menée par 0 la droite FOK parallèle au plan XM. Donc puisque le cercle roule, comme je l'ai montré à l'instant, la circonférence et la parallèle FK se couperont en des points variables, et les arcs compris entre la parallèle et le point de contact (par exemple LR) seront toujours égaux à l'arc BO. Et parce que la direction de l'impulsion demeure toujours la même qu'au commencement, l'impulsion poussera le cercle, pendant tout le roulement, suivant les droites parallèles à la première DOE, par exemple PLS, qui passent par les points d'intersection de la parallèle FK et de la circonférence, et déterminent dans le plan jusqu'au contact des droites égales à la première EB, par exemple SR. Pendant tout le temps du roulement l'impulsion dirige donc successivement tous les points de la circonférence, lorsqu'ils ont atteint la parallèle FK, c'est-à-dire lorsqu'ils sont distants du contact présent d'un arc égal à $\mathrm{OB}$, vers des points du plan variables (par exemple vers $S$ ) toujours distants du point de contact présent d'un intervalle égal à $\mathrm{EB}$. Mais cette direction constante et jamais interrompue suppose que le point 0 descend vers $\mathrm{E}$, et $\mathrm{L}$ vers $\mathrm{S}$, et ainsi de tous.

En effet quoique dans l'instant la poussée dirige immédiatement le point 0 vers le point $\mathrm{E}$, médiatement pourtant 0 est dirigé continuellement. Soit en effet un instant quelconque du roulement, par exemple celui où le cercle, de $B$, sera parvenu en $R$, coupant la parallèle FK en un autre point de sa circonférence L. À ce moment donc l'impulsion, comme montré plus haut, agit selon la droite PLS, parallèle à la première DOE, et par suite dirige le point $\mathrm{L}$ en $\mathrm{S}$; et par conséquent, comme autrement le point $\mathrm{L}$ ne peut se porter en $\mathrm{S}$, et que ni la gravité de la sphère soutenue par le plan, ni aucune inégalité du plan lui-même ne font opposition à un tel roulement, le cercle tend à rouler de telle façon que l'arc RL, qui est égal à BO, parcourt en tournant RS égal à BE. Et ainsi pendant tout le temps du roulement l'impulsion tend à ce que les arcs égaux à $\mathrm{BO}$ parcourent des espaces égaux à BE. Et parce que cela ne peut se faire sans que l'arc BO parcoure aussi la droite BE (car dans un roulement uniforme, le rapport d'un arc à l'espace qu'il parcourt est toujours égal à celui de l'autre arc à l'espace qu'il parcourt), il est manifeste que le point 0 , même après qu'il descend sous la parallèle $\mathrm{FK}$, alors que des points intermédiaires de la circonférence toujours différents descendent vers la parallèle, est dirigé continûment par l'impulsion vers E. C'est pourquoi comme rien n'empêche que le point 0 ne se porte en E, ni la solidité du plan - la conversion du cercle autour de son centre y pourvoit -, ni l'aspérité du plan, car par hypothèse il est parfait, ni la gravité du cercle ou de la sphère, car il est parfaitement soutenu par le plan, l'impulsion porte le point 0 en E, et par suite l'arc BO tourné circulairement parcourra la droite BE. Mais le rapport de l'arc BO à la droite qu'il parcourt en une fois est le même que celui de la circonférence entière à la droite parcourue par elle en une fois. Donc le rapport de la circonférence tournée une fois à la droite qu'elle parcourt est le même que celui de l'arc BO à la droite BE. Ce qui était proposé.

\section{Corollaire}

71 Donc il est aussi naturel à la sphère ou au cercle d'être portés sur un plan matériel parfait qui les soutient en roulant que sans rouler; et s'ils tournent, de parcourir en un roulement une ligne supérieure ou inférieure qu'égale à leur circonférence. Le fait est que 
la détermination en dépend de la direction de l'impulsion, comme on l'a démontré dans le Théorème.

\section{Théorème $\mathrm{XVI}$}

Un cercle reposant au point B sur un plan matériel parfait qui le soutient, poussé dans une direction définie, parcourra naturellement un espace plus grand que sa circonférence d'autant qu'on le veut.

Soit dans la circonférence un point quelconque $\mathrm{C}$, par lequel on fait passer OP parallèle au plan BE. Menons par ce point une autre droite NCE, qui coupe le plan en E. EB peut être plus grand que l'arc BC dans n'importe quelle proportion. Si l'on pousse le cercle suivant la droite NCE, par le Théorème XV, le rapport de BE à l'arc BC est le même que celui de l'espace accompli par le cercle en un tour à la circonférence du cercle. Donc l'espace parcouru par le cercle en un tour peut aussi être plus grand que la circonférence en proportion quelconque. Ce qui était proposé.

\section{Théorème XVII}

Et la sphère pourra être poussée de sorte qu'en un roulement, elle parcourra sur le plan un espace plus petit que sa circonférence. Pourtant jamais une impulsion droite ne la poussera de façon qu'elle parcoure un espace deux fois plus petit que sa circonférence.

Planche 18, fig. 8

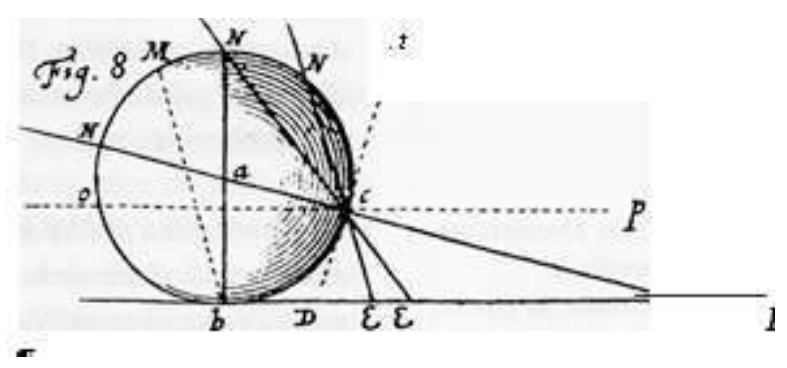

Soit un cercle poussé selon une droite quelconque coupant le cercle en $\mathrm{N}$ et $\mathrm{C}$, et le plan en $\mathrm{E}$. Soit ensuite la droite IC tangente au cercle en $C$, qui coupe le plan en D. Les tangentes $\mathrm{BD}$ et $\mathrm{CD}$ sont égales et prises ensemble seront toujours plus grandes que l'arc $B C$, quand bien même l'excès serait plus petit que toute grandeur donnée, si les points de contact se rapprochent toujours plus l'un de l'autre. Donc BD est aussi toujours plus grand que la moitié de l'arc BC. Donc BE sera aussi toujours plus grand que la moitié de l'arc $\mathrm{BC}$. Mais par le Théorème XV, la raison de $\mathrm{BE}$ à l'arc $\mathrm{BC}$ est la même que celle de l'espace parcouru par le cercle en un tour à la circonférence. Donc l'espace parcouru par le cercle en un tour sera aussi toujours plus grand que la moitié de la circonférence.

\section{Théorème XVIII}

Soit une sphère soutenue en $B$ par un plan matériel par fait et touchée par en dessous par un second plan $\mathrm{DZ}$, géométrique et non matériel. Si la sphère est poussée obliquement vers le haut selon une droite EGD qui coupe le plan supérieur 
en $D$, mais avec une force insuffisante pour soulever la sphère, celle-ci tournera de façon que la rotation s'effectue de $C$ vers $O$ et la translation de $B$ vers $X$ et $D$. Et le rapport de l'espace parcouru en un roulement à la circonférence sera égal à celui de la droite CD à l'arc CG.

Preuve de la première partie. Parce que l'impulsion s'effectue vers le haut selon la droite EGD, le mouvement imprimé par le moteur dirige le point $G$ vers le point $D$. Mais puisque par hypothèse le mouvement ne peut soulever la sphère, le point $G$ ne peut se diriger vers $\mathrm{D}$, à moins que la sphère ne tourne de $\mathrm{C}$ en 0 , et en même temps ne progresse vers $\mathrm{X}, \mathrm{D}$. C'est pourquoi, comme rien n'empêche ni cette rotation de la sphère autour de son centre de $C$ vers $0, n$ i sa translation vers $D, X$ (par hypothèse en effet la sphère repose sur un plan parfait dont le soutien supprime entièrement sa gravité), l'impulsion, pour diriger $\mathrm{G}$ en $\mathrm{D}$, en faisant tourner la sphère de $\mathrm{C}$ en 0 , la fait avancer vers $\mathrm{X}$, D. C'était le premier point.

Deuxième partie. Une démonstration semblable à celle de la deuxième partie du Théorème XV permettra de l'établir. Je puis en effet quitter à présent la plume. C'est pourquoi je laisse le lecteur appliquer les Théorèmes XVI et XVII à l'impulsion directe vers le haut. Nous étudierons ensuite les lignes décrites par les différents points d'un cercle qui roule ellesmêmes, et les espaces qu'elles enferment, ainsi que quelques autres phénomènes relatifs aux roulements.

\section{Théorème $\mathrm{XIX}$}

Lorsqu'un cercle roule sur un plan, le point de contact décrit une ligne courbe que nous appelons cycloide. Les autres points de la circonférence décrivent des cycloides égales et semblables, mais dont les parties sont différemment ordonnées.

Considérons le cercle en sa première situation 2. 3. 4. 5, et qu'il commence à rouler. Quand il parvient en E, le point de contact 2 est en $0:$ mais lorsque le roulement est parvenu en K, 2 sera en L. Et ainsi 2 descendra enfin par T en P. Il est donc évident que le point de contact $Z$ décrit la ligne courbe cycloïde.

82 Ensuite le point 5 , en $\mathrm{Z}$ dans la première position du cercle, a la même situation que le point de contact 2, lorsqu'il est placé en 0 . Donc la ligne OLTP que le point 2 placé en 0 décrit pendant que le cercle roule de $\mathrm{E}$ en $\mathrm{P}$, le point 5 la décrit pendant que le cercle roule de A en Q, savoir la ligne $\mathrm{ZNQF}$. Mais alors le point 5 sera en $\mathrm{Q}$. Mais 5 en $\mathrm{Q}$ se comporte de la même façon que 2 en A. Donc 5 situé en $Q$, pendant que le cercle continue de rouler de $Q$ en $P$, décrit une ligne courbe $Q X$ pareille à celle que décrit le point 2 pendant que le cercle roule de $\mathrm{A}$ en $\mathrm{E}$, savoir la ligne AO. Mais OLTP ajoutée à AO forme la totalité de la cycloïde AOLTP. Donc ZFNQ, avec QX, forme aussi une cycloïde égale et semblable à AOLTP. Avec le même raisonnement on prouvera la même chose pour les autres points de la circonférence.

83 On pourra tracer sur la figure jointe les chemins des différents points : 5 va de $Z$ en $Q$ par $\mathrm{F}$ et $\mathrm{N}$, et de $\mathrm{Q}$ en $\mathrm{X} .4$ est porté par $\mathrm{G}$ de $\mathrm{D}$ en $\mathrm{K}$, puis de $\mathrm{K}$ en a. 3 est porté par de $\mathrm{C}$ en $\mathrm{E}$, puis de $E$ en par $M$ et D'autre part du fait que la ligne parcourue par le cercle en un roulement, qui est la base de la cycloïde, peut être égale à la circonférence, mais aussi plus grande $o \mu$ plus petite d'autant qu'on le veut, résulte qu'il existe une infinité d'espèces de cycloïdes. Il est commode d'appeler primaire celle dont la base est égale à la circonférence du cercle générateur, et secondaires les autres. 

démonstration nouvelle et universelle de ce très célèbre théorème, conçue par nous même.

\section{Lemmes}

1. Si une droite EB touche des cercles égaux EG8, BVS, et si GK les coupe en retranchant des arcs égaux EG et $\mathrm{BK}$, la sécante GK sera parallèle à la tangente $\mathrm{EB}$. On le démontrera par les propositions 27 et 32 du Livre III, et par la $28^{e}$ du Livre I.

2. Si CB touche des cercles égaux, et si la parallèle IK est placée entre les circonférences des cercles, IK sera égale à la tangente $\mathrm{CB}$. On le démontre par la $27^{e} d u$ Livre III et les $28^{e}$ et $34^{e} d u$ Livre I.

3. Dans la progression arithmétique des nombres impairs, selon la suite naturelle qui commence par l'unité, 1. 3. 5. 7. 9. 11, etc., les extrêmes ou ceux qui sont également distants font deux fois le nombre des termes: 1 et 11 font 12, c'est-à-dire deux fois 6 , qui est le nombre des termes. De même 3 et 9 font 12. Et ainsi de suite. La raison en est que dans une somme quelconque il y a autant de binaires que de termes dans la progression.

\section{Théorème $X X^{12}$}

Soit une demi-cycloïde quelconque FTS, la moitié de sa base FB et sa hauteur BS, autour de laquelle on pose la moitié du cercle générateur BOUS.

Je dis que l'espace mixtiligne compris entre la cycloïde primaire STF, la base FB et la circonférence BOUS est double du demi-cercle générateur BUSA.

Dans les autres cycloïdes le mixtiligne en question à la même raison au demi-cercle générateur que le double de la base FB à la circonférence BOUS.

Soient ensuite tracés des demi-cercles égaux au générateur BOUS, tangents à la base aux points $\mathrm{C}, \mathrm{D}$, E, et qui coupent la cycloïde aux points $\mathrm{P}, \mathrm{M}, \mathrm{G}$.

91 Par la génération de la cycloïde, le rapport de EF qui a été parcouru par l'arc EG, à l'arc EG est comme celui de FB parcouru par la demi-circonférence EG $\theta$ ou BUS à la demicirconférence EG $\theta$ ou BUS. Donc en permutant le rapport de EF à FB est comme celui de l'arc EG à la demi-circonférence EG8 ou BUS. Mais par construction le rapport de EF à FB est aussi comme celui de l'arc BK à la demi-circonférence BUS. Donc les arcs EG et BK sont égaux. Je montrerai de même que les arcs DM et BO, et les arcs CP et BR sont égaux. Donc si on mène les droites $\mathrm{GKL}, \mathrm{MO}$ et $\mathrm{PQR}$, elles seront parallèles à la tangente $\mathrm{FB}$; et par suite chacun des segments interceptés, $\mathrm{KI}, \mathrm{IH}, \mathrm{HG}$, et $\mathrm{ON}$, NM, et RP sera égal aux tangentes $\mathrm{BC}, \mathrm{CD}, \mathrm{DE}$, ou bien à une seule d'entre elles, $\mathrm{BC}$. 
$\mathrm{EF}, \mathrm{KI}, \mathrm{IH}$ et $\mathrm{HG}, \mathrm{ON}, \mathrm{NM}$ et $\mathrm{RP}$, toutes égales à la même $\mathrm{BC}$, on l'a montré plus haut. La hauteur commune des huit triangles de la série supérieure PSR et de l'inférieure FGKB est $\mathrm{BL}$, car SQ et BL sont égales. Quant aux huit triangles des séries du milieu, la hauteur commune est LA, car QA et LA aussi sont égales. Donc puisque les bases de tous les triangles sont égales à la droite $\mathrm{BC}$, les huit triangles des séries supérieure et inférieure seront égaux au triangle de base octuple de BC et de hauteur BL. Et les huit triangles des séries PROM et GMOK seront égaux au triangle de base octuple de la droite BC et de hauteur LA. Donc tous ensemble, c'est-à-dire le rectiligne entier, sont égaux au triangle de base octuple de la droite $\mathrm{BC}$, et de hauteur égale au rayon $\mathrm{BA}$; c'est-à-dire à un triangle ayant une base double de FB, et de hauteur égale au rayon BA. Mais un tel triangle, si on considère la cycloïde primaire de base BF égale à la demi-circonférence BUS, est double du demi-cercle BUSA ${ }^{13}$; ou évidemment si ce n'est pas la cycloïde primaire qu'on considère, mais une qui a une base plus grande ou plus petite que la circonférence BUS, le rapport de ce triangle au demi-cercle sera comme celui du double de FB à la circonférence BUS $^{14}$. Donc le rectiligne, dans la cycloïde primaire, est double du demi-cercle BUSA ; et dans les autres cycloïdes, il a la même raison au demi-cercle que le double de BF à la circonférence BUS. Et avec le même discours exactement, nous le démontrerons pour tous les autres rectilignes de ce genre, quel que soit le nombre des côtés inscrits dans le cercle et la cycloïde, à condition que le nombre des divisions soit pair. Voilà une propriété toute merveilleuse des cycloïdes.

95 C'est pourquoi lorsque les côtés inscrits au cercle et à la cycloïde s'évanouissent enfin dans la circonférence BUS et la cycloïde FTS, et lorsque du coup le rectiligne finit par se transformer en mixtiligne, ce mixtiligne aussi sera double de la cycloïde primaire ; dans le cas des autres cycloïdes il aura la même raison au demi-cercle que le double de BF à la demi circonférence BUS. Ce qu'il fallait démontrer.

Je passe la réduction à l'impossible qui est à la portée d'un géomètre même médiocre.

97 Conséquence évidente. Donc l'espace cycloïdal primaire est triple du cercle générateur. Et les espaces cycloïdaux secondaires ont la même raison à leur cercle générateur que deux fois la base plus la circonférence à la même circonférence.

Courrier du Centre international Blaise Pascal, 14 | 1992 


\section{Scholie}

Depuis la première édition de cette dissertation, quelqu'un a taxé d'erreur la fin de cette démonstration, et déclaré que j'étais tombé dans le même paralogisme que j'avais reproché à Galilée dans le Scholie du Théorème I. Mais il en est tout autrement. Car lorsque j'ai dit que les côtés inscrits dans le cercle et la cycloïde finissent par s'évanouir dans la circonférence BUS et la cycloïde FTS, et que le rectiligne finit par se transformer en mixitiligne, j'ai seulement voulu dire que les espaces FG, GM, et MP, M S ainsi que BK, KO, OR et RS deviennent plus petits qu'un espace donné ; et que, de là, en supposant la première démonstration, la réduction à l'absurde est à portée; je laissais la démonstration aux lecteurs géomètres par souci de brièveté. Néanmoins, pour contenter tout le monde, j'ajoute ci dessous cette réduction à l'impossible.

Si l'on nie que le mixtiligne FTSUB est au demi-cercle BUSA dans le rapport de deux fois FB à la circonférence BUS, ce rapport sera ou plus grand ou plus petit. Supposons-le d'abord plus grand. Il y aura donc une autre quantité $\omega$ plus petite que le mixtiligne, par exemple d'un défaut $\delta$, qui sera au demi-cercle BUSA dans le rapport de deux fois FB à la circonférence BUS. Inscrivons dans le demi-cercle des côtés égaux, et autant de correspondants dans la cycloïde, selon le mode de construction employé dans la démonstration, de sorte que les espaces FG, GM, MP, et PS deviennent plus petits que $\delta$, ce dont je montrerai plus bas que c'est possible. Donc l'espace compris entre la périphérie BUS, la base FB et les côtés SP, PM, MG et GF sera plus grand que. $\omega$ Il en résulte que le rectiligne compris entre les côtés $\mathrm{SR}, \mathrm{RO}$, OK et $\mathrm{KB}$, la base $\mathrm{FB}$ et les côtés SP, PM, MG, et GF sera beaucoup plus grande que $\omega$. Mais on avait voulu que le rapport de $\omega$ au cercle BUSA soit comme celui du double de FB à la circonférence BUS. Donc la raison du mixtiligne au demi-cercle est plus grande que deux fois BF à BUS : ce qui contredit les démonstrations précédentes.

Supposons à présent, s'il est possible, que le mixtiligne FTSUB ait au demi-cercle BUSA une raison plus petite que deux fois BS à BUS. Donc inversement le demi-cercle a une plus grande raison au mixtiligne que BUS à deux fois FB. Donc la raison du demi-cercle est à une quantité quelconque $\lambda$ plus grande que le mixtiligne d'un excès $\zeta$ par exemple, comme BUS à deux fois FB. Inscrivons dans le demi-cercle autant de côtés égaux, de sorte que les espaces BK, KO, OR et RS deviennent plus petits que $\zeta$ (ce que fait Euclide, 2, 12) et complétons le rectiligne comme ci-dessus. L' espace compris entre les côtés SR, RO, OK, $\mathrm{KB}$, la base BF et la cycloïde FTS est plus petit que $\lambda$; donc puisque l'on voulait que le demi-cercle soit à $\lambda$ dans le même rapport que BUS à deux fois $\mathrm{FB}$, le demi-cercle sera à l'espace compris par les côtés $\mathrm{SR}, \mathrm{RO}, \mathrm{OK}, \mathrm{KB}$, la base $\mathrm{FB}$ et la cycloïde, en plus grande raison que BUS à deux fois $\mathrm{FB}$. Donc le demi-cercle est au rectiligne compris entre les côtés SR, RO, OK, KB, la base FB et les côtés GF, FM, MP et PS en une beaucoup plus grande raison que BUS à deux BF. Ce qui contredit de nouveau ce qui a été démontré plus haut.

Je montre comme suit que les segments FG, GM, MT, PX et PS finissent par devenir plus petits qu'un espace donné. Divisons $\mathrm{FB}$ en tant de parties égales que le rectangle $\mathrm{CB}$ devienne enfin plus petit qu'un espace donné ; achevons le rectiligne, comme ci-dessus, avec autant de côtés inscrits au cercle qu'à la cycloïde ; menons le diamètre $C \delta \mathrm{du}$ demicercle $\mathrm{CN} \delta$, qui coupe BUS en $\alpha$ et $\beta$; et joignons $\mathrm{S} \delta$. Si des demi-cercles égaux SOB et $\delta \mathrm{NC}$ on relève $\alpha \beta O$ qui leur est commun, et si aux restes on ajoute les espaces mixtes $S \alpha \delta$ et $\mathrm{B} \beta C$, l'espace $S \delta \mathrm{NCBOUS}$ sera égal au rectangle $\mathrm{CBS} \delta$, donc plus petit que l'espace donné. 
Ensuite le petit segment $\pi \mathrm{S}$ est plus petit que le segment $\mathrm{SR}$, c'est-à-dire que le segment PN. Il est donc clair que l'espace entier S $\delta$ NCBUS compris entre les droites $S \delta, \mathrm{BC}$ et les circonférences BUS et $\mathrm{CN} \delta$, est plus grand que le segment cycloïdique P $\pi S P$. C'est pourquoi puisqu'on a montré que l'espace $\delta \delta$ NCBUS est plus petit que le donné, P $\pi S P$ le sera aussi. Je montrerai de la même façon que chacun des segments restants PM, MG et GF devient aussi plus petit que le donné. Il est donc manifeste que tous pris ensemble seront enfin plus petits que le donné.

\section{Lemme pour le théorème suivant}

Que le cercle BFAQ passe par le centre A du plus grand cercle BLHD, qu'il touche intérieurement. Si on mène du centre $A$ la droite $A Q R$, elle coupera des arcs égaux $B Q$ et $B R$. Du centre $Z$ du petit cercle menons $Z Q$, et soit la parallèle $A \alpha$. Les arcs $\alpha B$ et $Q B$ seront évidemment semblables, car ils sous-tendent des angles au centre égaux $\mathrm{BA} \alpha, \mathrm{BZQ}^{15}$. Aussi comme les circonférences entières BLHD et BFAQ sont en raison double l'une de l'autre, l'arc $\mathrm{B} \alpha$ sera double de $\mathrm{QB}^{16}$. Ensuite puisque ${ }^{17}$ l'angle au centre $\mathrm{BZQ}$ est double de l'angle $\mathrm{BAQ}, \mathrm{BA} \alpha$, égal aussi à BZQ, sera double de l'angle BAQ, ou BAR. Donc ${ }^{18}$ l'arc $\alpha \mathrm{B}$ est aussi double de l'arc BR. Par conséquent les arcs $\mathrm{QB}$ et BR sont égaux. Ce qu'il fallait démontrer.

On démontrera la même chose en menant la tangente $\mathrm{AD}$ et la droite $\mathrm{OQ}$, et encore d'une autre façon si du point $\mathrm{H}$ on mène la parallèle à $\mathrm{AC}$.

Mais on le démontre aussi sans tracer aucune ligne à partir des données du lemme. Puisque l'angle au centre est double de l'angle à la circonférence du même arc ou d'un arc semblable, le même angle BAC à la circonférence du petit cercle BFAC et au centre du grand BLHD coupera un arc BR, semblable à la moitié de BQ. Mais un arc quelconque du grand cercle BLHD est double du semblable dans le petit cercle: ils sont en effet semblables, dans le rapport des circonférences. Donc l'arc BR est double de la moitié de $B Q$. Donc il est égal à BQ entière.

Fig. 9

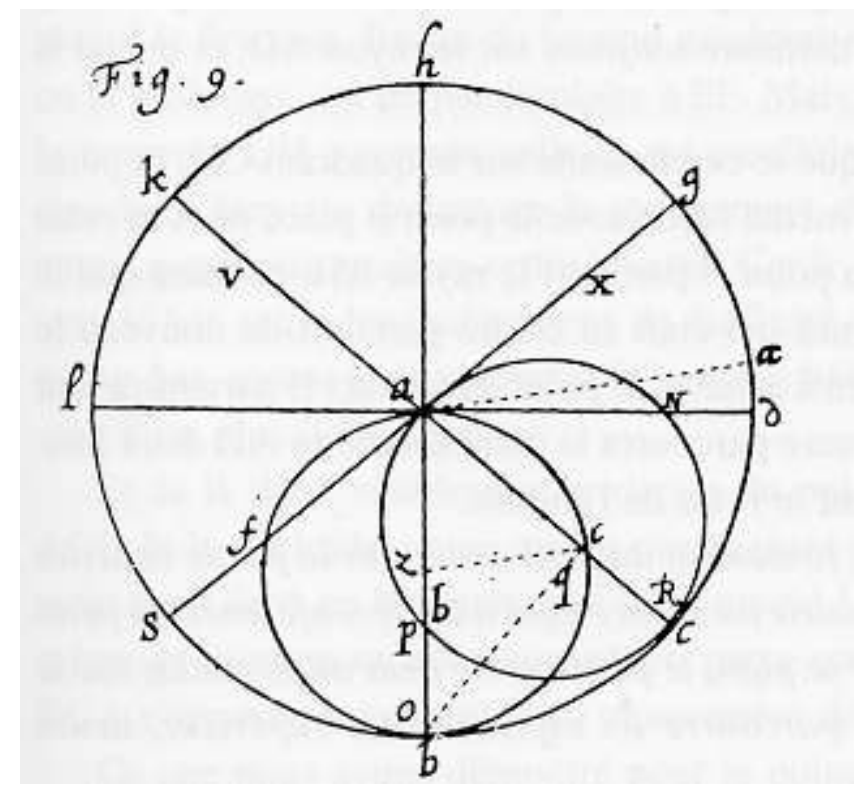




\section{Théorème XXI}

Soit le cercle BLHD, touché intérieurement par un petit cercle BFAC, qui passe par son centre $A$. Pendant le temps où le petit cercle roule une fois vers $D$, sur la concavité de la circonférence du grand cercle, les points de la circonférence du petit décrivent des diamètres qui passent par ces points.

Le point de contact $\mathrm{B}$ parcourt le diamètre $\mathrm{BH}^{19}$.

Le point de contact qui est au centre A parcourt deux fois le rayon AD, en allant de $A$ à $D$, puis en revenant de $D$ en $A$.

Le point $F$ (tout comme les autres points de la demi-circonférence BFA) monte d'abord sur la partie FG du diamètre, puis redescend sur l'espace $\mathrm{GX}$, qui est égal à FS. Le point $Q$ (tout comme n'importe quel autre point de la demi-circonférence $B Q A)$ descend d'abord le long de la partie $Q R$ du diamètre, puis revient sur le diamètre jusqu'en $\mathrm{V}$, laissant la partie $\mathrm{VK}$ égale à $\mathrm{QR}$. Le centre $\mathrm{Z}$ décrit la circonférence dont le rayon est $\mathrm{AZ}$.

Prenons dans la circonférence du petit cercle un point quelconque $C$, et du centre A par C ou $Q$ tirons une droite qui coupe la circonférence du grand cercle en R. Par le lemme démontré ci-dessus, les arcs $\mathrm{OQ}$ et $\mathrm{OR}$ sont égaux. C'est pourquoi si le cercle $\mathrm{AQBF}$ roule de 0 en $R$, le point $R$ descendant à partir de $Q$ arrivera en $R$; mais le cercle $A Q B F$ coupera alors $A B$ en $P$ : et de nouveau, par le lemme, les arcs RP et RO seront égaux. Nécessairement le point $B$, qui était d'abord en 0 , se trouve alors en $P$, lorsque de 0 le roulement est parvenu en $R$. Je montrerai de la même façon, quelle que soit la partie du roulement parcourue dans le quadrant $\mathrm{BD}$, que le point $\mathrm{B}$ est toujours en un point $\mathrm{du}$ rayon OA.

Mais à présent, dans le même temps que le point $\mathrm{B}$ venant de 0 parcourt le demi diamètre $\mathrm{OA}$, le point qui est au centre $\mathrm{A}$ parcourt le rayon $\mathrm{AD}$; je le montre ainsi. Puisque le diamètre $\mathrm{HO}$ est double du rayon $\mathrm{AO}$, la circonférence HDBL est aussi double de la circonférence ACBF. Donc le quadrant BD du grand cercle est égal à la moitié de la circonférence du petit ACO. Et par le lemme les arcs OQ et OR sont égaux. Donc les restes $\mathrm{QA}$ et $\mathrm{RD}$ sont égaux aussi. Mais par le lemme RD est égal à RN. Donc QA et RN sont égaux. Mais par le lemme l'arc $O Q$ est aussi égal à l'arc $O R$, c'est-à-dire par le lemme à l'arc RP. Donc si aux égaux QA et RN on ajoute les égaux OQ et RP, les touts OQA et PRN seront égaux. Donc lorsque le roulement est parvenu en $\mathrm{R}$, et que le point $\mathrm{B}$ est venu de $0 \mathrm{en} \mathrm{P}$, le point qui était au centre $\mathrm{A}$, a été mû de $\mathrm{A}$ jusqu'au point $\mathrm{N}$, qui se trouve sur le rayon $\mathrm{AD}$. De la même façon je montrerai que ce point demeure toujours sur le rayon $A D$, et quand $B$ atteindra le centre A, l'autre sera en D.

111 En outre, comme je l'ai montré, pendant que le cercle roule sur le quadrant OD, le point qui est au centre parcourt le rayon $\mathrm{AD}$; de la même façon avec le point $\mathrm{B}$ placé en $\mathrm{A}$ et celui qui était en A placé en $\mathrm{D}$, on montrera que le point $\mathrm{B}$ parcourt le rayon $\mathrm{AH}$, pendant que le cercle roule sur le quadrant $\mathrm{DH}$; mais le point qui était au Centre parcourt de nouveau le demi-diamètre de $\mathrm{D}$ en $\mathrm{A}$. Et, un roulement ainsi achevé, le point de contact $\mathrm{B}$ parcourra tout le diamètre $\mathrm{OAH}$. Mais le point qui est au centre parcourra le demidiamètre $\mathrm{AD}$ deux fois.

112 À partir de cela, on démontrera facilement le reste de l'énoncé. 
113 Il faut entendre seulement ce théorème du roulement du petit cercle sur la partie concave de la circonférence du grand qui lui fait parcourir un espace égal à la circonférence du petit. En effet, comme nous l'avons démontré pour le plan, le petit cercle peut aussi rouler sur le grand de telle façon qu'en un roulement il parcoure un espace tantôt supérieur, tantôt inférieur à sa circonférence.

\section{Théorème XXII}

Quoique la sphère soit le plus régulier de tous les corps, quand elle est roulée sur un plan avec une vitesse uniforme, ses parties se meuvent nécessairement avec une vitesse irrégulière.

Planche 18, fig. 6

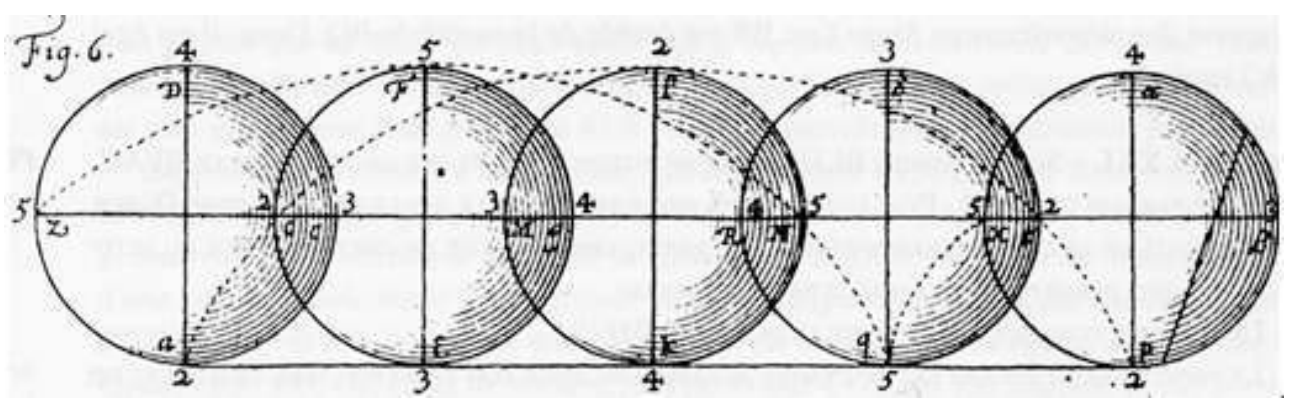

115 Coupons le grand cercle d'une sphère en quatre quadrants aux points $2,3,4,5$. Soit AP la longueur que la sphère parcourt en un roulement, égale à sa circonférence. Si elle est divisée en quatre parties égales aux points $\mathrm{E}, \mathrm{K}$ et $\mathrm{Q}$, chacune de ses parties sera égale à chacun des quadrants de la circonférence. Puisque donc le quadrant AC est égal à la droite $\mathrm{AE}$, dans le même temps que la sphère roule de $\mathrm{A}$ en $\mathrm{E}, 3$ se porte de $\mathrm{C}$ en $\mathrm{E}$ et 2 de $\mathrm{A}$ en 0 ; et par suite A parcourt la courbe AO. D'autre part, puisque EK est égale au quadrant EG, pendant le temps que la sphère roule de $E$ en $K, 4$ se porte de $G$ en $K$, et ainsi 2, diamétralement opposé à 4 , se porte de 0 en $\mathrm{L}$, parcourant la courbe OL. Mais par hypothèse la sphère roule uniformément, c'est-à-dire par la première définition, parcourt les espaces égaux $\mathrm{AE}$ et $\mathrm{EK}$ en des temps égaux. Donc le point 2 aussi parcourt en des temps égaux les courbes AO et OL. Mais OL est beaucoup plus grande que AO. Donc selon la définition 2 , le point 2 se meut de $\mathrm{A}$ en $\mathrm{L}$ avec une vitesse irrégulière.

116 Mais il faut chercher plus profond la raison de ce phénomène. Pendant que la sphère roule sur le plan, le point de contact 2 ou A est porté sur la cycloïde ALP par un certain mouvement composé de deux mouvements, dont l'un est droit tendant de A en P, l'autre circulaire de A vers Z, suivant la circonférence AZDC. C'est pourquoi pendant que la sphère tourne de $A$ vers $\mathrm{P}$, emportant avec elle le point $\mathrm{A}$, celui-ci parcourt entretemps la circonférence AZDC, mouvement qui affecte le premier inégalement.

Car lors de la montée suivant le demi-cercle AZD, le premier fait certainement opposition, jusqu'en un certain point, mais de moins en moins : ensuite par la partie qui reste du même demi-cercle, il aide de plus en plus continuellement. Et en descendant suivant le demicercle DCA, les mêmes choses arrivent, mais en ordre inverse.

En effet le mouvement ascendant sur la demi-circonférence AZD s'effectue suivant l'infinité des différences des inclinaisons des tangentes en nombre infini qu'on peut mener en chaque point de la demi-circonférence : plus celles-ci touchent en un point 
élevé, moins le mouvement qu'elles déterminent vers le haut s'oppose au mouvement droit de A vers F, ou plus il le favorise. En fin du second quadrant AZ, il est porté suivant la tangente $\mathrm{ZB}$, qui, si on la prolonge, est perpendiculaire à EF. Mais en fin du second quadrant, il est porté suivant la tangente $\mathrm{DH}$; comme celle-ci est parallèle à $\mathrm{EF}$, il est évident qu'en $\mathrm{D}$ le mouvement circulaire favorise davantage le mouvement droit de $\mathrm{A}$ vers $\mathrm{F}$. Il en va de même dans les autres quadrants, mais en ordre inverse. Car le mouvement descendant suivant DCA se porte vers le bas selon les inclinaisons de l'infinité des tangentes; plus celles-ci touchent en des points bas, moins le mouvement fait vers le bas selon elles favorise le mouvement droit de A vers F ou plus il s'y oppose.

Et de là il est visible que la vitesse du point 2 croît continuellement sur toute la moitié AOL de la cycloïde, parce que le mouvement circulaire s'oppose toujours moins au mouvement droit de $\mathrm{A}$ en $\mathrm{K}$; mais sur l'autre moitié LIP la vitesse diminue continuellement dans la même proportion qu'elle augmentait, parce que le mouvement circulaire sur le demi-cercle DCA s'oppose toujours plus au mouvement de A en P.

Ce que nous avons démontré pour le point de contact, nous le démontrerons en mêmes termes de n'importe quels autres points donnés dans la sphère.

Mais on demandera peut-être en quel point de la circonférence le mouvement circulaire commence à favoriser le droit et cesse de s'y opposer. La résolution de cette question n'est ni étrangère à notre propos ni indigne de conclure notre dissertation sur le roulement du cercle. Soit donc ce

\section{Lemme}

On donne un parallélogramme FC. Si le côté BF est porté d'un mouvement uniforme sur $\mathrm{BC}$ jusqu'à ce qu'il coïncide avec le côté $\mathrm{CE}$, de sorte que dans le mouvement il demeure toujours parallèle à $\mathrm{CE}$, et que dans le même temps le point $\mathrm{B}$ parcoure le côté $\mathrm{BE}$ d'un mouvement uniforme, je dis que le point $B$ décrit le diamètre $B E$.

Ce lemme est d'Aristote, dans les Questions mécaniques ${ }^{20}$. Moi je le démontre ainsi. Posons que le côté BF soit parvenu en AM qui coupe le diamètre en $\mathrm{O}$; et soit menée par $\mathrm{O}$ la parallèle $\mathrm{GN}$ à $\mathrm{BC}$. Puisque par hypothèse les mouvements sont uniformes, la proportion des vitesses de chaque mouvement sera semblable à celle des espaces accomplis dans les mêmes temps par chaque mouvement. Donc le rapport de $\mathrm{BC}$, c'est-àdire l'espace que le côté $\mathrm{BF}$ parcourt dans le temps entier, à BF ou CE, espace parcouru par le point $\mathrm{B}$ dans le même temps, est égal au rapport de $\mathrm{BA}$, que parcourt le côté $\mathrm{BF}$ en une partie du temps, à la longueur que le point $B$ parcourt dans la même partie du temps. Mais cette dernière est $\mathrm{BG}$ ou $\mathrm{AO}$; car la raison de $\mathrm{BC}$ à $\mathrm{BF}$, c'est-à-dire $\mathrm{CE}$, est celle de $\mathrm{BA}$ à $\mathrm{AO}$. Donc quand le côté $\mathrm{BF}$ parvient en $\mathrm{MA}$, le point $\mathrm{B}$ est en 0 , qui est un point du diamètre $\mathrm{BE}$. Je montrerai de même pour tout autre instant de ce mouvement que le point $B$ est sur le diamètre. La proposition est donc évidente. 
Planche 18, fig. 14.

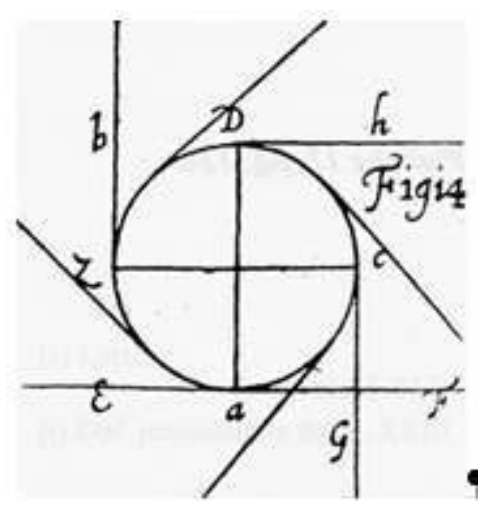

Planche 17, fig. 123.

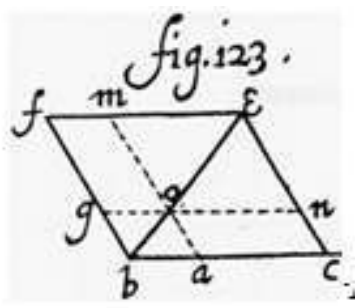

Définition

Un mouvement est dit s'opposer à un autre lorsque le mouvement composé par les deux parcourt un espace moindre que chacun d'eux parcourrait seul dans le même temps; il le favorise si cet espace est plus grand ${ }^{21}$.

\section{Théorème XXIII}

Soit le cercle EGDQ tangent au plan en $E$; dans le temps qu'il est porté vers $M$, le point $\mathrm{E}$ monte par la circonférence EGD, et ensuite descend par DQ en E. Que d'autre part le mouvement droit et le mouvement circulaire soient également rapides et uniformes. Qu'ensuite, vers le haut de part et d'autre du point de contact, on coupe $E G$ et $E Q$, sixièmes parties de la circonférence entière. Je dis qu'aux points $G$ et $Q$ le mouvement circulaire ne favorise pas le mouvement droit ni ne s'y oppose. 


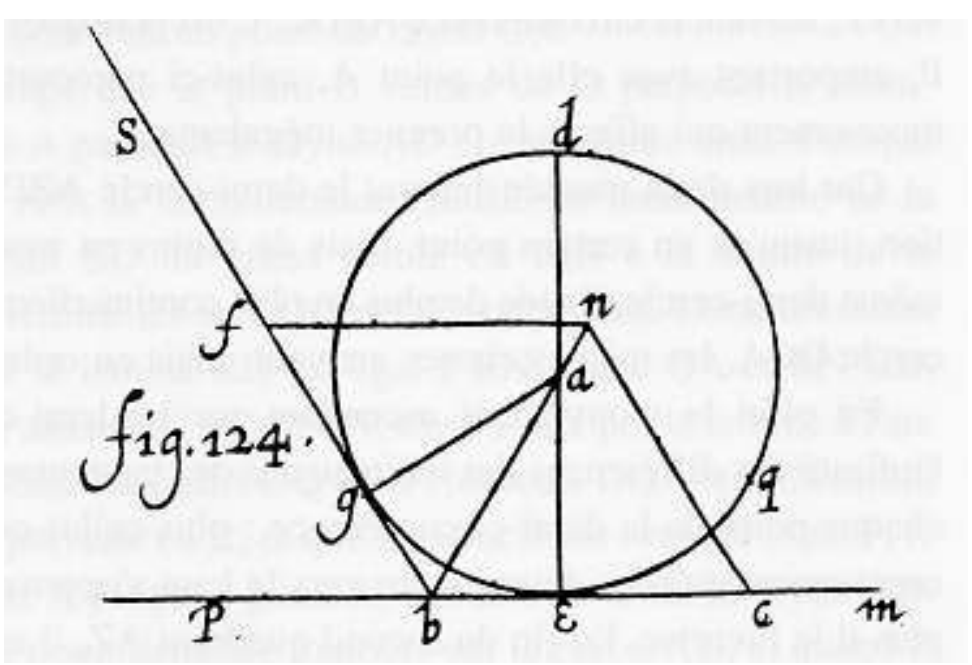

Soit $\mathrm{BS}$ la tangente en $\mathrm{G}$; on prend $\mathrm{BF}$ et $\mathrm{BC}$ quelconques égaux. Compléter le rhombe $F B C N$. Du centre $A$ du cercle, on mène $A B$, et les droites $A G$ et $A E$ aux points de contact $G$ et $E$. Puisque les tangentes $G B$ et $E B$ sont égales, ainsi que $A G$ et $A E$, et que $A B$ est commun, la droite BA coupe visiblement l'angle GBE en deux parties égales. Puisque BA prolongée passe par $\mathrm{N}$, c'est aussi le diamètre du rhombe. Mais les quatre angles du quadrilatère GAEB pris ensemble sont égaux à quatre droits. C'est pourquoi ${ }^{22}$ comme les deux angles AGB et AEB sont deux droits, les restes GAE et GBE font ensemble deux droits. Mais GAE fait deux sixièmes de deux droits, puisque l'arc EG est le sixième de la circonférence. Donc GBE fait quatre sixièmes de deux droits. Donc puisque BA est bissectrice de GBE, GBN et NBC font chacun deux sixièmes de deux droits. Mais puisque $\mathrm{NC}$ et $\mathrm{BF}$ sont parallèles, $\mathrm{CNB}$ est égal à GBN. Donc CNB aussi fait deux sixièmes de deux droits. C'est pourquoi comme j'ai montré que NBC fait deux sixièmes, il est évident que le reste $\mathrm{BCN}$ aussi fait deux sixièmes. Donc $\mathrm{CNB}$ et $\mathrm{BCN}$ sont égaux et ainsi le diamètre $\mathrm{BN}$ du rhombe est égal au côté $\mathrm{BC}^{23}$.

Mais à présent quand le point $\mathrm{E}$, montant le long de la circonférence est arrivé en $\mathrm{G}$, il est porté selon l'inclinaison de la tangente BGF ; et cela, par hypothèse, avec la même vitesse qu'il progresse en même temps vers $\mathrm{M}$ d'un mouvement droit. Mais le mouvement qui suit l'inclinaison de la tangente BGF ne favorise pas le mouvement d'égale vitesse par la tangente $\mathrm{BC}$ et ne s'y oppose pas : je le montre ainsi.

Puisque le mouvement par lequel le point $\mathrm{B}$ avance sur BF et celui par lequel il est porté sur $B C$ sont d'égale vitesse, pendant le temps que le point $B$ parcourrait $B F$, le déplacement du point $\mathrm{B}$ accomplirait $\mathrm{BC}$ égal à $\mathrm{BF}$. Mais le point $\mathrm{B}^{24}$ par ce mouvement double décrit $\mathrm{BN}$, diamètre du rhombe $\mathrm{FC}$. C'est pourquoi comme on a démontré plus haut que $\mathrm{BN}$ est égal à $\mathrm{BC}$, le mouvement composé du mouvement de translation vers $\mathrm{M}$ et du mouvement d'égale vitesse suivant l'inclinaison de la tangente $\mathrm{BF}$ accomplit une longueur $\mathrm{BN}$ égale à celle - égale à $\mathrm{BC}$ - parcourue par le seul mouvement de translation sur $\mathrm{BC}$. C'est pourquoi le mouvement selon l'inclinaison de la tangente BGF ne favorise pas le mouvement vers $\mathrm{M}^{25}$ et ne s'y oppose pas. Puisque quand le point $\mathrm{E}$ s'élève par la circonférence EGD, à la fin de l'arc EG il est porté selon la tangente BGF ; il est évident que le mouvement circulaire en $G$ ne s'oppose pas au mouvement droit de $E$ vers $C$ ni ne le 
favorise. D'où il en va aussi évidemment de même pour le point Q. La proposition est donc évidente.

\section{Théorème XXIV}

130 Avec les mêmes données, je dis que le mouvement circulaire s'oppose au mouvement droit à partir de $\mathrm{E}$ jusqu'à $\mathrm{J}$, c'est-à-dire sur le sixième de la circonférence; qu'il le favorise ensuite sur quatre sixièmes de la circonférence, GDQ; et qu'il ne s'y oppose derechef que sur le sixième de la circonférence restant QE.

Soit pris dans l'arc EG un point quelconque K, auquel BKS touche le cercle. En retranchant de part et d'autre les portions égales $\mathrm{BF}$ et $\mathrm{BC}$, qu'on complète le rhombe $\mathrm{FNCB}$, et qu'on mène du centre $A$ la droite $A B$. De même, qu'on trace les droites $A K$ et $A E$ aux contacts $K$ et $\mathrm{E}$. On a montré dans le précédent que la droite $\mathrm{AB}$ est bissectrice de l'angle $\mathrm{KBE}$, et passe par l'angle $\mathrm{N}$ du rhombe ; de même les angles KBE et KAE font deux droits. Puisque l'arc EK est moindre que la sixième partie de la circonférence, l'angle KAE sera moindre que le sixième de quatre droits, c'est-à-dire deux sixièmes de deux droits. Donc KBE est plus grand que quatre sixièmes de deux droits : et par conséquent NBK d'une part et NBC d'autre part sont plus grands que deux sixièmes de deux droits. Mais comme NC et BF sont parallèles, BNC est égal à NBK. Donc BNC est aussi plus grand que deux sixièmes de deux droits. C'est pourquoi comme NBC est aussi plus grand que deux sixièmes, le reste $\mathrm{BCN}$ est plus petit que deux sixièmes ; et par conséquent plus petit que BNC. Donc BC est plus grand que le diamètre du rhombe BN.

Maintenant lorsque le point E montant circulairement est parvenu en K, il est porté suivant l'inclinaison de la tangente BKF, et parce qu'en même temps il va vers $\mathrm{M}$ avec la même vitesse, pendant le temps que le mouvement par BKF met à parcourir la droite BF, le mouvement par BM d'égale vitesse parcourt $\mathrm{BC}$ égal à $\mathrm{BF}$. Mais $\mathrm{BN}$ est le chemin du mouvement composé de ces deux ${ }^{26} ; \mathrm{j}^{\prime}$ ai montré déjà qu'il est plus petit que la droite $\mathrm{BC}$, qui aurait été parcourue par le mouvement droit seul. Donc le mouvement selon l'inclinaison de la tangente BKF, c'est-à-dire le mouvement circulaire même au point $\mathrm{K}$ s'oppose $^{27}$ au mouvement vers $\mathrm{M}$ : par suite le mouvement circulaire s'oppose à la translation vers M sur tout l'arc EG. C'était le premier point; il est évident qu'il en va aussi de même sur l'arc QE. 


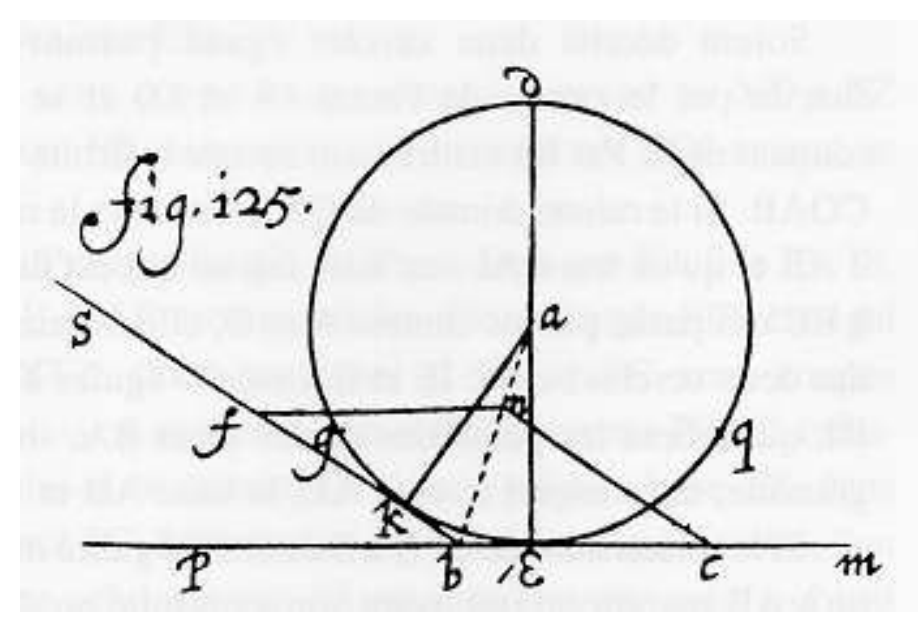

Soit ensuite pris au-delà de la sixième partie de la circonférence EG, entre $G$ et le sommet $\mathrm{D}$, un point quelconque K. Étant menée la tangente BKS, soient pris de part et d'autre BF et $B C$ égaux; on complète le rhombe BFNC. On mène ensuite du centre $A$, comme auparavant, les droites $\mathrm{AK}, \mathrm{AB}$ et $\mathrm{AE}$. Puisqu'alors l'arc $\mathrm{EK}$ est plus grand que le sixième de la circonférence, l'angle EAK sera plus grand que le sixième de quatre droits, donc que deux sixièmes de deux droits. Il en résulte que KBE est moindre que quatre sixièmes de deux droits, et par suite $A B K$ et $A B E$ qui sont égaux, sont chacun plus petits que deux sixièmes de deux droits. Mais comme $\mathrm{CN}$ et $\mathrm{BF}$ sont parallèles, BNC est égal à $\mathrm{ABK}$. Donc $B N C$ est aussi moindre que deux sixièmes. C'est pourquoi comme $A B C$ aussi est moindre que deux sixièmes, le reste $\mathrm{BCN}$ est plus grand que deux sixièmes, et par suite plus grand que BNC. En découle que BC est plus petit que le diamètre du rhombe BN. Cependant BN est le chemin ${ }^{28} \mathrm{du}$ mouvement composé par le mouvement selon l'inclinaison de la tangente BKF et par le mouvement de translation vers $\mathrm{M}$, de vitesse égale; mais $\mathrm{BC}$ est le chemin parcouru par le mouvement de translation vers $\mathrm{M}$ à lui seul, toutes choses évidentes par la première partie. Donc le mouvement selon l'inclinaison de la tangente BKF, c'est-à-dire le mouvement circulaire même au point $K$, favorise le mouvement vers $M^{29}$. C'est pourquoi, le point $K$ ayant été choisi quelconque dans l'arc GD, il est évident que le mouvement circulaire favorise le mouvement droit vers $\mathrm{M}$ tout au long de l'arc GD. Il est clair qu'il en va de même pour l'arc DQ. 


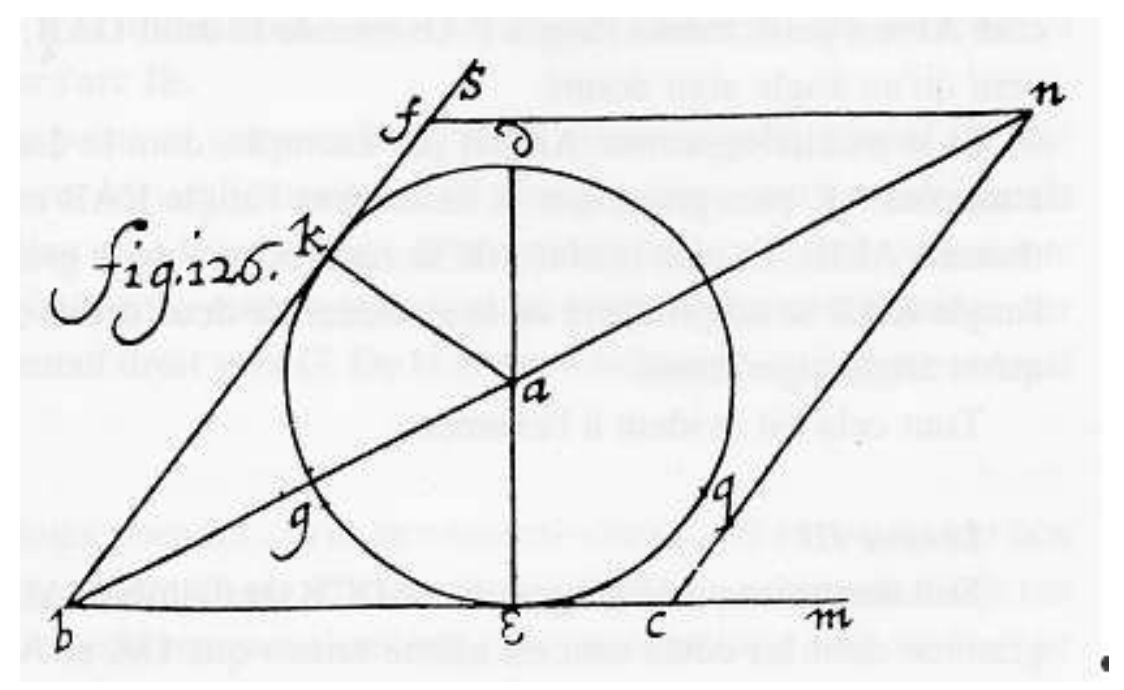

\section{Lemme I}

Construire un parallélogramme de diamètre $(\mathrm{AH})$ égal à la base $(\mathrm{AB})$; et dont le côté (AF) soit à la base $(\mathrm{AB})$ en raison donnée ( $\mathrm{Q}$ à $\mathrm{S}$ ).

Soient décrits deux cercles égaux passant chacun par le centre de l'autre (A et $\mathrm{O}$ ) et se coupant en E. Par les centres soit menée la droite COAB. Si la raison donnée de Q à $S$ est alors la raison d'égalité, qu'on mène $\mathrm{AE}$, $\mathrm{El}$ parallèle à $\mathrm{AB}$ et qu'on trace $\mathrm{AI}$ : on aura fait ce qui est demandé. En effet, parce que IE est parallèle à $B C$ qui passe par les centres $A$ et 0 , elle est aussi parallèle à DN qui est tangente à chacun des deux cercles; donc IE et $\mathrm{BA}$ sont ${ }^{30}$ égales à $\mathrm{DN}$, donc entre elles. C'est pourquoi $\mathrm{AE}$ et $\mathrm{BI}$, qui relient les parallèles égales $\mathrm{IE}$ et $\mathrm{BA}$, sont parallèles. Donc AEIB est un parallélogramme, dans lequel le côté $\mathrm{AE}$, la base $\mathrm{AB}$ et le diamètre $\mathrm{Al}$ sont égaux.

Si la raison donnée de $Q$ à $S$ était d'inégalité moindre, que $Q$ soit à $S$ dans le même rapport qu'à $\mathrm{AB}$ une droite qui ayant son extrémité en $\mathrm{A}$ est inscrite dans le cercle $\mathrm{ANC}$, soit $\mathrm{AF}$; qu'après avoir mené $\mathrm{FH}$ parallèle à $\mathrm{AB}$, on joigne les droites $\mathrm{AH}$ et $\mathrm{BH}$. Je montrerai, comme plus haut, que $\mathrm{AFHB}$ est un parallélogramme dans lequel $\mathrm{FA}$ a, avec $\mathrm{AB}$, une raison d'inégalité donnée moindre, et que le diamètre $\mathrm{AH}$ est égal à la base $\mathrm{AB}$.

Enfin si la raison donnée de $Q$ à $S$ est d'inégalité supérieure, il faudra qu'elle soit moindre que la raison double. Que le rapport de $Q$ à $S$ soit celui d'une certaine droite à $A B$. Elle sera selon la condition posée ci-dessus, plus petite que $\mathrm{CA}$ double de $\mathrm{AB}$; et par suite elle pourra être inscrite au cercle $A N C$, comme c'est le cas pour $A K$ Menons KG parallèle à $A B$ et traçons $B G$ et $A G$. Je montrerai de nouveau comme ci-dessus que AKGB est un parallélogramme, dans lequel le côté $A K$ est plus grand que la base $A B$ en raison donnée, mais que le diamètre $A G$ et la base $A B$ sont égaux. La démonstration de la détermination est évidente. 


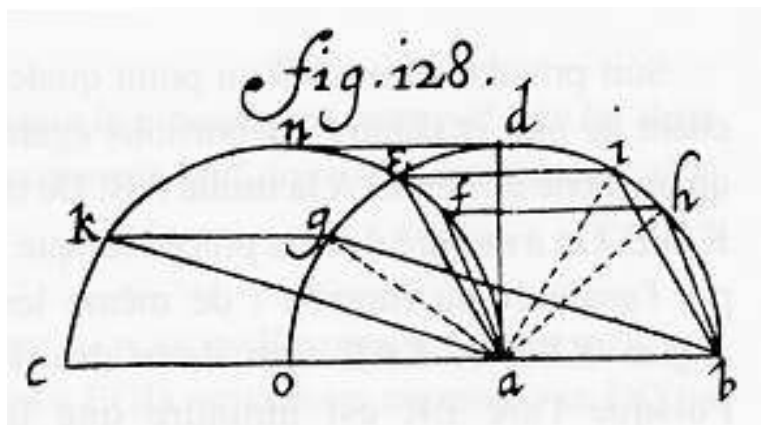

\section{Lemme II}

Le parallélogramme AFHB par exemple, dont le diamètre $\mathrm{AH}$ est égal à la base $\mathrm{AB}$, mais dont le côté $A F$ est plus petit que la base, a un angle $F A B$ plus petit que l'angle $E A B$ du rhombe $\mathrm{AEIB}$, dans lequel la base $\mathrm{AB}$, le diamètre $\mathrm{AI}$ et le côté $\mathrm{AE}$ sont égaux. Et plus le côté $\mathrm{AF}$ est petit, moins l'angle $\mathrm{FAB}$ excède le droit $\mathrm{DAB}$, et cet excès deviendra enfin plus petit qu'un angle aigu donné.

Si le parallélogramme $A K G B$ par exemple, dont le diamètre $A G$ est égal à la base $A B$, a un côté $A K$ plus grand que la base, alors l'angle $K A B$ est plus grand que l'angle $E A B$ du rhombe AEIB. Et plus le côté $\mathrm{AK}$ se rapprochera de la grandeur de $\mathrm{AC}$, double de $\mathrm{AB}$, plus l'angle $\mathrm{KAB}$ se rapprochera de la grandeur de deux droits; et à la fin le défaut sera moindre qu'un angle aigu donné.

Tout cela est évident à l'examen.

Planche 17, Fig. 129

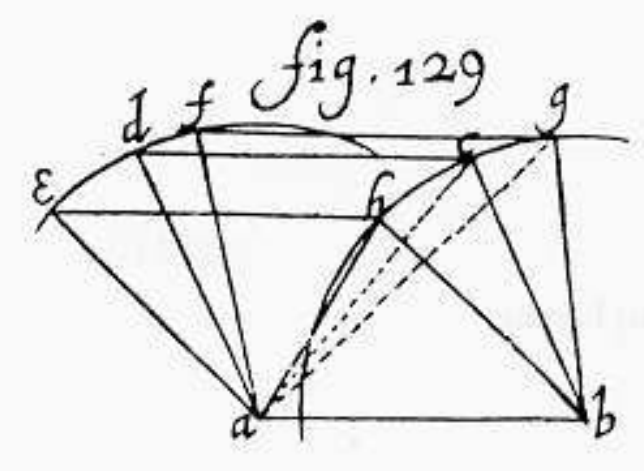

\section{Lemme III}

Soit donné un parallélogramme $\mathrm{ADCB}$ de diamètre $\mathrm{AC}$ égal à la base $\mathrm{AB}$. Le parallélogramme dont les côtés sont en même raison que $\mathrm{DA}$ et $\mathrm{AB}$, s'il a un angle plus grand que l'angle $\mathrm{DAB}$, a un diamètre moindre que sa base ; s'il a un angle plus petit que $\mathrm{DAB}$, son diamètre est plus grand que sa base.

Avec A et B pour centres, on décrit des cercles par D et C, et on trace le parallélogramme dont les angles $\mathrm{E}, \mathrm{H}, \mathrm{F}$ et $\mathrm{G}$ se trouvent sur les circonférences. Leurs côtés seront 
proportionnels à $\mathrm{DA}$ et $\mathrm{BA}$, c'est-à-dire égaux : et le diamètre $\mathrm{AH}$ du parallélogramme qui a l'angle $\mathrm{EAB}$ plus grand que l'angle $\mathrm{DAB}$, est plus petit que la droite $\mathrm{AC}$, c'est-à-dire que la base $A B$. Mais le diamètre $A G$ de l'autre parallélogramme qui a l'angle $F A B$ plus petit que l'angle $\mathrm{DAB}$, est plus grand que la base $\mathrm{AB}$. Mais la raison des diamètres aux bases dans les parallélogrammes $A E H B$ et $A F G B$ est semblable à celle qui se trouve dans tous les autres qui leur sont semblables. La proposition est donc évidente.

\section{Théorème XXV}

Soient les mêmes données qu'au théorème XXIII ; mais que le mouvement circulaire EGDQE soit maintenant plus lent que le mouvement droit vers $M$.

Soit ${ }^{31}$ le parrallélogramme XZ de diamètre TV égal à la base TZ; mais que le côté XT soit inférieur à la base TZ dans la même proportion que le mouvement circulaire est plus lent que le droit. Que du centre on mène alors AR, qui fasse avec la tengeante PEC un angle ARC égal à l'angle XTZ. Qu'on mène sur AR la perpendiculaire AK qui coupe le cerle en $\mathrm{K}$; et qu'on retranche l'arc IE égal à l'arc EK.

Je dis que le mouvement circulaire plus lent que le droit dans le rapport de XT à TZ s'oppose au mouvement droit sur tout l'arc EK, mais qu'il le favorise sur KDI et que de nouveau il s'y oppose sur IE.

Soit en effet menée la tangente SKP ${ }^{32}$. Puisque l'angle AHP est droit, par Euclide III, 18, et que KAR est droit par construction, SKP et AR sont parallèles. Donc l'angle EPS est égal à l'angle CRA; c'est-à-dire à l'angle XTZ. C'est pourquoi si PS et PC sont coupés proportionnellement aux côtés XT et $\mathrm{TZ}$, et si on complète le parallélogramme PSNC, celui-ci sera semblable à XZ; puisque dans celui-ci le diamètre TV est égal à la base TZ, dans l'autre le diamètre PN est aussi égal à la base PC. Maintenant s'il y a deux mouvements, l'un suivant la droite PS, l'autre par PC, tels que celui qui suit PS est plus lent que celui qui suit PC dans la même raison que SP est plus petit que PC, dans le même temps l'un parcourra PS et l'autre PC. Et par suite ${ }^{33}$ le chemin du mouvement composé des deux accompli dans le même temps sera le diamètre $\mathrm{PN}$, diamètre du parallélogramme. Parce que ce chemin est égal ${ }^{34}$ à la base PC, qui est parcourue par le mouvement vers $C$, il est évident que le mouvement selon l'inclinaison de la tangente PS ne s'oppose pas au mouvement de translation par PC. C'est pourquoi comme le mouvement circulaire EKD suit au point K l'inclinaison de la tangente PKS, celui-ci ne s'oppose pas à la translation droite vers $\mathrm{C}$, ni ne la favorise ${ }^{35}$. D'où il en va évidemment aussi de même pour le point $\mathrm{I}$.

Soit ensuite pris un point quelconque a dans l'arc EK ; que la droite $\omega \alpha$ y soit tangente : s'il y a dans l'angle $\alpha \omega C$ un parallélogramme dont les côtés soient proportionnels à SP et $\mathrm{PC}$, son diamètre est ${ }^{36}$ plus petit que la base; mais ce diamètre est le chemin ${ }^{37}$ que parcourt le mouvement composé par le mouvement suivant l'inclinaison de la tangente $\omega \alpha$, et du mouvement qui parcourt la base dans le même temps et tend vers $C$. Il est donc évident que le mouvement suivant l'inclinaison de la tangente $\omega \alpha$, c'est-à-dire le mouvement circulaire au point $\alpha$ s'oppose $^{38}$ à la translation vers $\mathrm{C}$. Donc puisque le point $\alpha$ a été pris quelconque le mouvement circulaire s'oppose à la translation vers $\mathrm{C}$ sur tout l'arc EK. De là il en va évidemment de même aussi pour l'arc IE.

Soit ensuite pris entre $\mathrm{K}$ et le point au sommet $\mathrm{D}$ un point quelconque $\theta$, auquel est tangente $\delta \theta$; et dans l'angle $\delta \theta \mathrm{E}$, tracer le parallélogramme ${ }^{39}$ dont les côtés sont 
proportionnels à SP et PC; son diamètre ${ }^{40}$ est plus grand que la base. D'où, en appliquant les raisonnements précédents, il est facile de voir que le mouvement circulaire au point $\theta$, et par suite aussi sur tout l'arc KD, favorise le mouvement droit vers $C$. De là il en va évidemment de même aussi pour l'arc DI.

\section{Théorème XXVI}

Avec les mêmes données : Si le mouvement circulaire est plus lent que le droit, le circulaire s'oppose au droit de part et d'autre sur des arcs plus grands que les sixièmes EG et $\mathrm{QE}$. Et plus le mouvement circulaire est lent, plus grands sont de part et d'autre les arcs sur lesquels il s'oppose au mouvement droit: mais ils sont toujours plus petits que les quadrants EG et $\mathrm{HE}$, si lent que soit le mouvement circulaire.

Soit la même construction que dans les précédents, où nous montrons, en supposant le mouvement circulaire inférieur au droit comme XT à TZ, c'est-à-dire ${ }^{41}$ comme SP à PC ; que le circulaire s'oppose au droit sur l'arc EK, et en descendant sur l'autre partie sur l'arc IE, égal à EK. Mais par la démonstration du Théorème XXIII, il est évident que, si de la droite $B \beta$, tangente au point $G$, extrémité de la sixième partie $E G$, et de la tangente $B M$ on retranche des grandeurs égales, et si on complète le rhombe, son diamètre est égal à la base. Donc dans le parallélogramme PSNC de diamètre PN égal à la base PC, mais dont le côté SP est plus petit que la base, l'angle SPC est plus petit ${ }^{42}$ que l'angle GBC du rhombe. Donc le point $\mathrm{K}$ où le côté $\mathrm{SP}$ touche le cercle est nécessairement plus haut que $\mathrm{G}$, où le côté du rhombe est tangent, et par suite l'arc EK est plus grand que le sixième EG. C'était le premier point.

Ensuite, plus le mouvement circulaire est inférieur au droit, plus aussi (comme c'est visible par la construction précédente) SP doit être inférieur à PC. Donc l'angle SPC devient aussi d'autant plus petit ${ }^{43}$ que l'angle $\mathrm{GBC}$ du rhombe, et par suite le contact $\mathrm{K}$ est d'autant plus élevé, et l'arc EK d'autant plus grand. C'était le second point.

Enfin parce que l'angle du parallélogramme de diamètre égal à sa base demeure toujours ${ }^{44}$ plus grand qu'un droit, il est évident que le côté SP, dont le contact détermine la grandeur de l'arc sur lequel le mouvement circulaire s'oppose au droit, ne peut être tangent en $O$, extrémité du quadrant EO, ou au-dessus de $O$. D'où paraît le troisième point. 


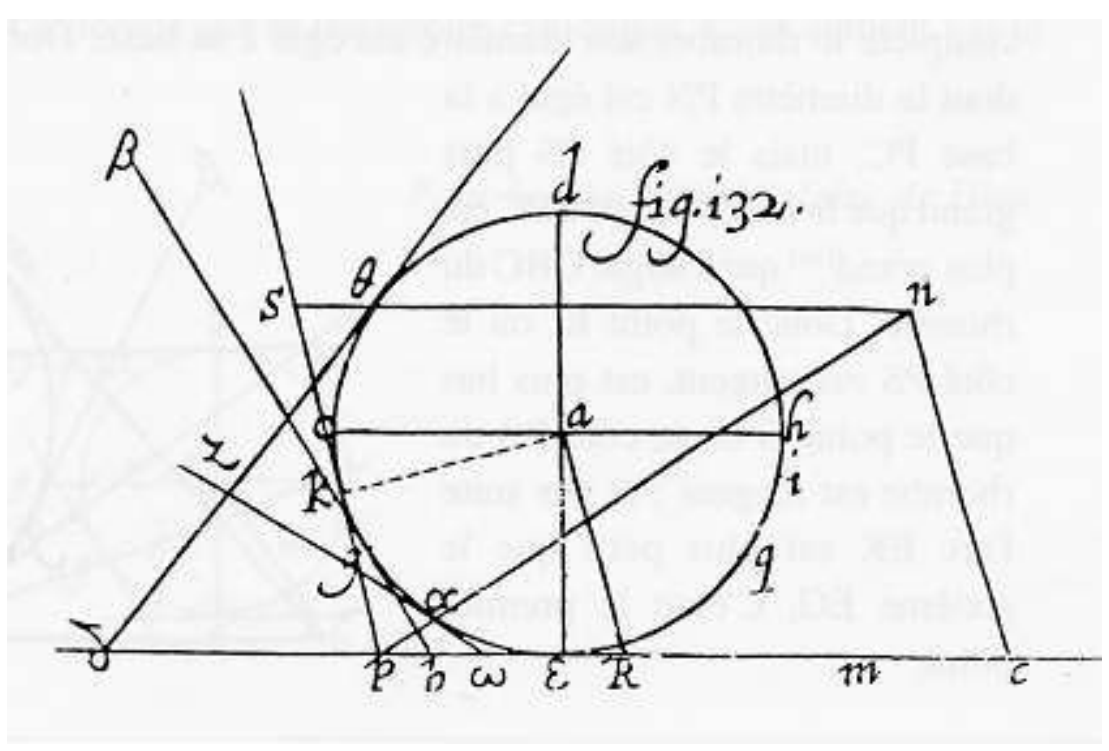

\section{Théorème XXVII}

Soient les mêmes données que ci-dessus ; mais que le mouvement circulaire soit à présent plus rapide que le mouvement de translation droit, quoique moindre que double. Soit le parallélogramme $\mathrm{XZ}^{45}$ de diamètre $\mathrm{TV}$ égal à la base $\mathrm{TZ}$; que le côté $\mathrm{XT}$ soit plus grand que la base TZ dans le même rapport que le mouvement circulaire est supposé plus rapide que le mouvement droit. Soit ensuite menée du centre du cercle AR, qui fasse avec la tangente EM l'angle ARM égal àl'angle XTZ. Menons alors AK perpendiculaire à AR, qui coupe le cercle en $\mathrm{K}$; et retranchons EI égal à l'arc EK. Je dis que le mouvement circulaire, plus rapide que le mouvement droit en même proportion que XT surpasse TZ, s'oppose au droit su l'arc EK ; mais qu'il le favorise sur KDI ; puis qu'en descendant il s'y oppose dérechef sur IE.

La démonstration est la même qu'au Théorème XXV.

\section{Théorème XXVIII}

Avec les mêmes données : Si le mouvement circulaire est plus rapide que le droit, mais moindre que double, il s'oppose de part et d'autre au droit sur les arcs plus petits que les sixièmes de circonférence $\mathrm{EG}$ et $\mathrm{QE}$, et plus il approche de la vitesse double, plus il s'oppose de part et d'autre sur des arcs plus petits, qui finissent par devenir plus petits que n'importe quelle grandeur.

Soit la même construction que dans le précédent ; et que SKP touche le cercle en K. Qu'on retranche $\mathrm{PS}$ et $\mathrm{PC}$, proportionnels à $\mathrm{TX}$ et $\mathrm{TZ}$, et qu'on complète le parallélogramme PSNC. Son côté $\mathrm{SP}$ a même raison à la base $\mathrm{PC}^{46}$ que le mouvement circulaire au droit, et le diamètre $\mathrm{PN}$ est égal à la base $\mathrm{PC}$. En effet $\mathrm{SC}$ est semblable à XZ, où TV est égal à TZ. Mais on a déjà démontré ci-dessus ${ }^{47}$ qu'en supposant que le mouvement circulaire surpasse le droit dans la même proportion que SP surpasse PC, le circulaire s'oppose au droit sur l'arc EK en montant, et sur l'arc égal IE en descendant. Ensuite, selon ce qui a été démontré dans le Théorème XXIII, il est évident que si de la droite B (qui touche le cercle en G, 
extrémité de la sixième partie $\mathrm{EG}$ ) et de la tangente $\mathrm{BM}$ on retranche des longueurs égales, et si on complète le rhombe, son diamètre est égal à sa base. Donc dans le parallélogramme PSNC dont le diamètre PN est égal à la base PC, mais le côté PS plus grand que la base, l'angle SPC est plus grand ${ }^{48}$ que l'angle GBC du rhombe. Donc le point $\mathrm{K}$, où le côté $P S$ est tangent, est plus bas que le point $G$ où le côté $B$ du rhombe est tangent; et par suite l'arc EK est plus petit que le sixième EG. C'était le premier point.

Planche 17, fig. 131

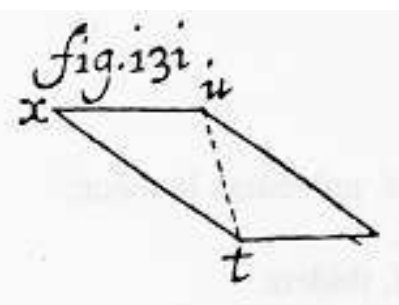

Ensuite plus le mouvement circulaire approche de la vitesse double, plus aussi en vertu de la construction le côté SP approche du double de la base PC. Donc l'angle SPC devient aussi ${ }^{49} \mathrm{~d}$ 'autant plus grand et approche plus de la grandeur de deux droits, et par suite le contact $\mathrm{K}$ en est d'autant plus bas et l'arc EK devient plus petit, et finit par devenir plus petit que tout donné : en effet l'angle SPC devient enfin inférieur à deux droits ${ }^{50}$ d'un angle qui devient plus petit que tout donné; il en découle nécessairement que l'arc EK devient enfin inférieur à tout donné. C'était le second point.

\section{Théorème XXIX}

Avec les mêmes données : si le mouvement circulaire EGDQE est plus rapide du double, ou plus, que le mouvement de translation droit vers $\mathrm{C}$, en aucun point de la circonférence il ne s'y opposera, mais il le favorisera totalement.

Planche 17, fig. 130

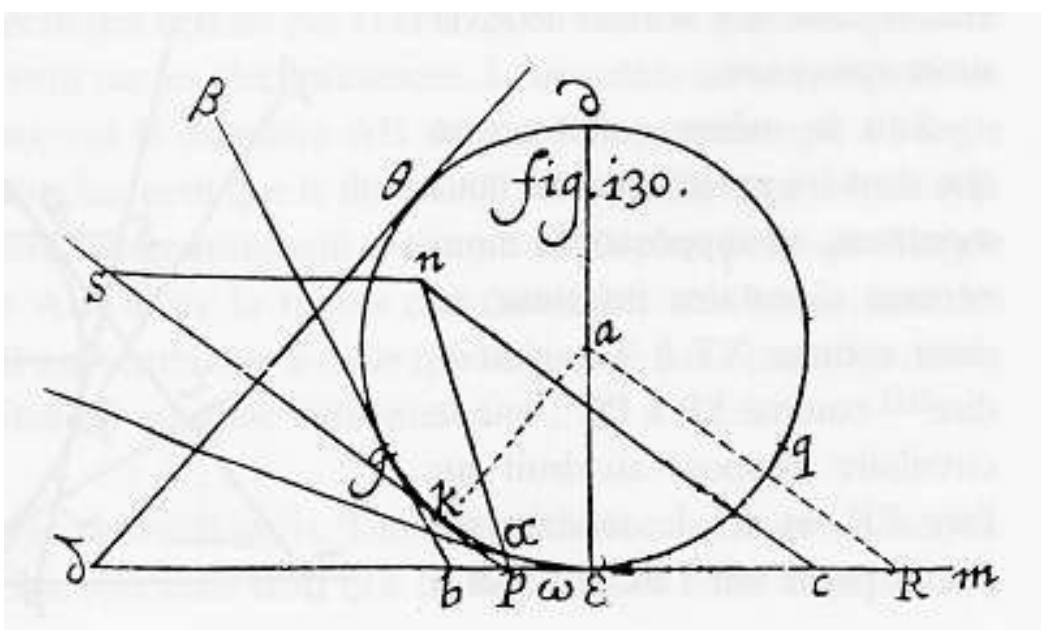

La démonstration est assez claire par la seconde partie du Théorème précédent. Néanmoins, au cas où cela paraîtrait utile, je le démontre ainsi. Posons d'abord ce postulat: deux mouvements circulaires de vitesses différentes ne peuvent s'opposer au même mouvement droit sur des parties égales de la circonférence. Que s'oppose à un 
mouvement droit, s'il est possible, un mouvement circulaire plus rapide du double ou plus, sur un certain arc EK. Soit menée la tangente SKP, et dans l'angle SPE soit le parallélogramme PSNC, de diamètre PN égal à la base PC. Il est manifeste par la démonstration du Théorème XXVII que le mouvement circulaire dont la vitesse est à celle du mouvement droit dans le rapport de SP à PC, s'oppose au mouvement droit sur l'arc EK. Mais le côté SP est nécessairement moindre que le double de la base PC, comme il est évident par la détermination du lemme 1 du Théorème XXV : et par suite le mouvement circulaire aussi, qui s'oppose au mouvement droit sur l'arc EK, est plus rapide que le mouvement droit de moins que le double. Donc deux mouvements circulaires inégaux, l'un plus rapide que le droit du double, l'autre plus rapide de moins que le double, s'opposent au même mouvement droit sur le même arc EK, ce qui contredit le postulat.

\section{Corollaire}

Par ce que nous avons démontré dans les sept derniers Théorèmes, on voit, lorsqu'une sphère roule sur un plan, combien le mouvement circulaire empêche ou favorise le mouvement d'un point quelconque dans la sphère progressant sur sa cycloïde. Si en effet le mouvement circulaire est aussi rapide que le droit (ce qui paraît toujours au regard lorsqu'une sphère roule librement sur un plan matériel), il empêche la progression $d u$ point sur sa cycloïde pendant un tiers du temps qui s'écoule pour un roulement complet; mais il l'aidera pendant les deux tiers du même temps. C'est visible par le Théorème XXIV.

Si le mouvement circulaire est plus lent que le droit, il empêche la progression du point sur sa cyclö̈de de plus que sur le tiers du temps d'un roulement entier ; mais il le favorise sur moins que les deux tiers du temps. Et plus le mouvement circulaire est surpassé par le droit, plus longtemps il l'empêche, mais toujours pendant moins que la moitié du temps. Cela est clair par le Théorème XXVI.

Si le mouvement circulaire est plus rapide que le droit (mais moins que du double), il l'empêche sur moins que le tiers du temps. C'est clair par le Théorème XXVIII.

Enfin s'il est plus rapide du double ou plus, il ne l'empêche plus du tout, mais favorise la progression du point pendant tout le temps sur sa trajectoire cycloïdale. C'est évident par le Théorème XXIX.

Pour la plus grande gloire de Dieu.

Fig.13

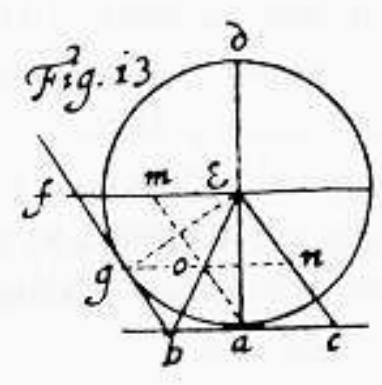




\section{NOTES}

1. Galilée, Discours concernant deux sciences nouvelles, éd. Clavelin, Paris, Colin, 1970, p. 125 sq.

2. Pour traduire le latin rasio, j'emploi ce terme, quoiqu'il soit inusité en ce sens, afin d'éviter l'équivoque avec le mot glissement. On verra au Théorème VIII les difficultés qui découlent de cette définition.

3. Galilée, Discours..., III, Théorème II, éd Clavelin, p. 127.

4. Ces références renvoient aux Eléments d'Euclide

5. Pris à la lettre, le raisonnement est faux, comme on peut le vérifier sur la figure. Mais cela ne change rien à la substance de la conclusion.

6. Aristote, Problèmes mécaniques, 24, 855 a ; voir trad. Hett, XIV, Minor Works, Loeb, W. Heinemann, London, 1980, p. 387 sq. Outre Aristote et Galilée, la Dissertation de 1650 mentionne Blancanus, c'est-à-dire Joseph Biancani, SJ, élève de Clavius et auteur d'un commentaire d'Aristote; mais le Père Tacquet note seulement que «Blancanus aliud non exhibet quam paraphrasim Aristotelis»(p.13). D'autre part l'avertissement Ad Lectorem de 1650 mentionne loannes Bapt. Benedictus (Benedetti), « aliique permulti », sans s'y arrêter davantage.

7. Galilée, Ibid., I, éd. Clavelin, p. 22 sq.

8. Galilée, Ibid., p. 43 sq. Le texte latin de la Dissertatio de 1651 porte: «quomodo maior circumferentia semel convoluta percurrat lineam se majorem »; il y a là un lapsus pour minorem. Galilée dit : "comment au cours d'une rotation du petit cercle, le plus grand décrit une ligne droite plus courte que sa circonférence ». L'Index erratorum ne corrige pas l'erreur.

9. Dans le Des Mouvements composés, Roberval distingue dans le mouvement de la Roulette deux mouvements, «l'un circulaire, par le moyen duquel le point (...) parcourt une fois la circonférence de son cercle, l'autre droit (...) «tant tous deux uniformes ». Il poursuit : "d'où vous voyez que ces deux mouvements étant uniformes, le point (...) peut décrire trois diverses sortes de Roulettes, suivant que son mouvement circulaire sera proportionné à son mouvement droit, ou si vous voulez suivant la raison de circonférence de son cercle à la ligne (...) que le centre décrit (...) » (p. 58 sq.). Je cite d'après Mémoires de l'Académie des Sciences depuis 1666 jusqu'à 1699, VI, Paris, 1730, Compagnie des Librairies.

10. Torricelli, De Dimensione Parabolae, Appendix de Dimensione Cycloïdis ? in Opere, I, Faenza, p. 163.

11. Voir les F. a Schooten Commentarii in Librum II, dans l'édition latine de la Géométrie de Descartes, note 0. L'étude originale de la cycloïde par Descartes se trouve dans sa lettre à Mersenne du 27 juillet 1638 (Mersenne, Correspondance, VII, p. 407 sq.) et la lettre à Mersenne du 23 août 1638 (Ibid ., VIII, p. 34 sq.). Les Éléments de Géométrie montrent que le P. Tacquet apprécie l'œuvre mathématique de Descartes.

12. Démonstration résumée par G. Maupin (voir bibliographie) pour le cas de la roulette simple.

13. Par Arch. p. l. dim. Circ.

14. 2. 1. 1. Cycl. \& Annul.

15. 29. 1 .

16. Pappus. 1. 5. P. 41.

17. 20.3.

18. 33. 6 .

19. Cas particulier de la construction de l'hypoclycloïde lorsque, le rayon du petit cercle étant moitié du rayon du grand, elle se confond avec le diamètre du grand cercle. 
20. Aristote, Problèmes mécaniques, 1, 848 b. On trouve un lemme semblable dans Roberval, Des Mouvements composés, éd. cit., p. 6-7, sans référence à Aristote.

21. On verra plus bas que l'aide et l'empêchement que le mouvement circulaire apporte à la translation sont mesurés par la distance parcourue par le point mû par ces deux mouvements, représentée d'après le $\mathrm{P}$. Tacquet par la diagonale du parallélogramme des mouvements. Selon, dit-il, que la diagonale est plus longue ou plus courte que la base horizontale du parallélogramme, qui correspond au mouvement de translation, on dira que le mouvement circulaire l'aide ou l'empêche. De là découlent les résultats auxquels le P. Tacquet parvient dans la fin de sa Dissertatio.

22. 18. Elem. 3.

23. 6.2 .

24. Lemme.

25. Défin.

26. Lemme.

27. Déf. Précédant le théor. XXIII.

28. Lemme.

29. Défin.

30. Dem. 2, Théor. 20.

31. Par le lem. I précéd.

32. Les Errata de 1651 indiquent que a lin. 16, c'est-à-dire dans la démonstration de ce théorème, citationes turbatae omnes igitur omissae censeantur. Nous laissons pourtant subsister ces références à titre documentaire...

33. Lemm. Théor. XXIII.

34. Par construction.

35. Lemme 3 précéd.

36. On le montre comme ci-dessus.

37. Lemm. I précéd.

38. Lemm. 3 précéd.

39. Def. Précédant le théor. XXIIII.

40. Def. Ibidem.

41. Par la constr. Précéd.

42. Lemm. 2. Théor. XXV.

43. Ibid.

44. Ibid.

45. Lem. I. Théor. XXV.

46. Constr. Précéd.

47. Théor. XXVII.

48. Lemm. 2. Théor. XXV.

49. Ibidem.

50. Ibidem.

\section{NOTES DE FIN}

a. 18.3 (4). 

b. 28.1.
c. 18.3
d. 28.1
e. Défin. 3
f. Théor.1

INDEX

Mots-clés : physique, mathématiques, cercle, roulement

Keywords : physics, mathematics, circle, rolling

\section{AUTEUR}

\section{PÈRE ANDRÉ TACQUET}

Professeur de Mathématiques au Collège de la Société de Jésus de Louvain. 